\title{
On The Use Of Models To Assess Foot-And-Mouth Disease Transmission And Control
}

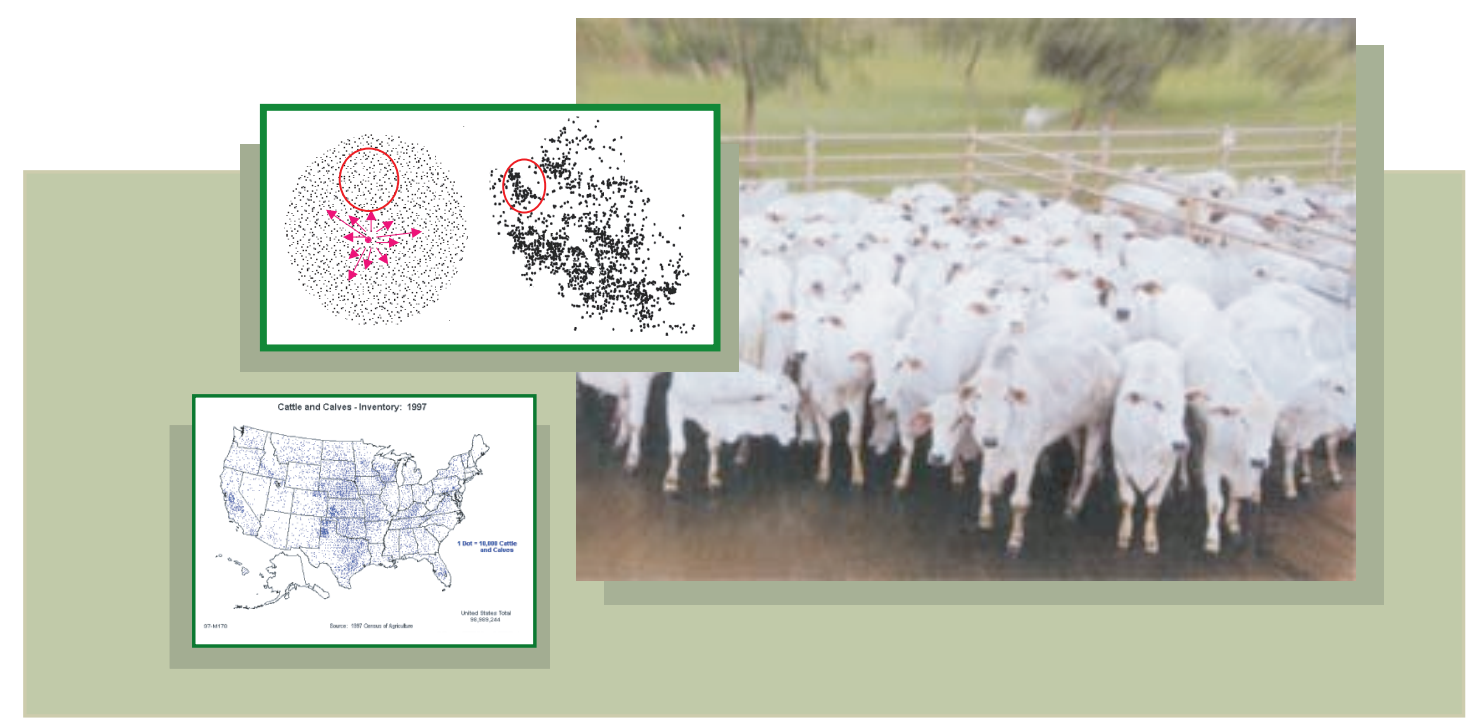

Author:

Tanya Kostova - Vassilevska, LLNL

Consultants:

Tom Bates (LLNL),

Mark Thurmond, Tim Carpenter, UC Davis

July 20, 2004

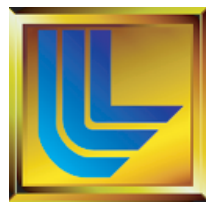

UCRL-TR-20524I

Lawrence Livermore National Laboratory

University of California • Livermore, California 94550 
This document was prepared as an account of work sponsored by an agency of the United States Government. Neither the United States Government nor the University of California nor any of their employees, makes any warranty, express or implied, or assumes any legal liability or responsibility for the accuracy, completeness, or usefulness of any information, apparatus, product, or process disclosed, or represents that its use would not infringe privately owned rights. Reference herein to any specific commercial product, process, or service by trade name, trademark, manufacturer, or otherwise, does not necessarily constitute or imply its endorsement, recommendation, or favoring by the United States Government or the University of California. The views and opinions of authors expressed herein do not necessarily state or reflect those of the United States Government or the University of California, and shall not be used for advertising or product endorsement purposes.

This work was performed under the auspices of the U.S. Department of Energy at the University of California Lawrence Livermore National Laboratory under contract No. W-7405-Eng-48. 


\section{TABLE OF CONTENTS}

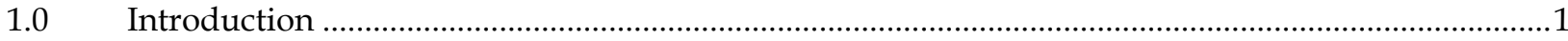

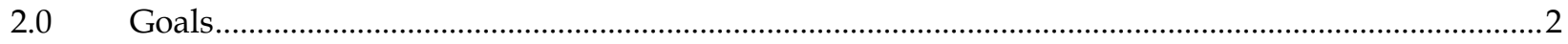

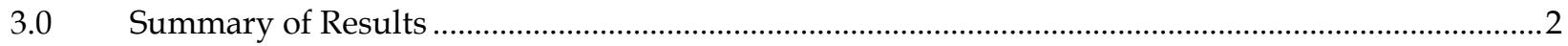

4.0 Background Information on Epidemic Models.................................................................................

4.1 The General Architecture Of Epidemic Models.......................................................................

4.2 The Bottom-Up and Top-Down Modeling Approaches .................................................................5

4.3 Types Of Epidemic Models With Regard To Spatial Detail ...........................................................

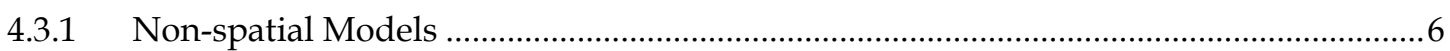

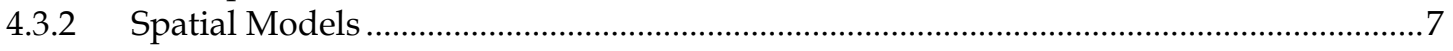

4.4 Types of Models with Regard to Purpose

4.4.1 Epidemic Models for Evaluating Control Policies ....................................................... 8

4.4.2 Economic (Cost/Benefit Evaluation) Models......................................................................

5.0 Modeling Disease Transmission Among Livestock Populations ........................................................

5.1 Factors for Disease Transmission in Livestock.......................................................................

5.2. Specifics of Foot and Mouth Disease Spread and Their Significance for Epidemic Modeling.10

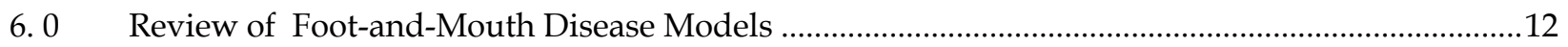

6.1. Intra-Herd (On-the-Farm) Models ...........................................................................................12

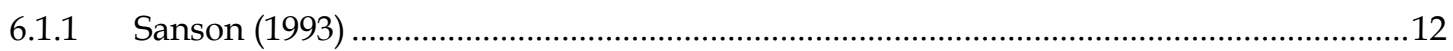

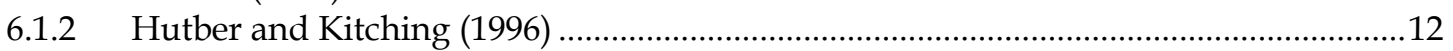

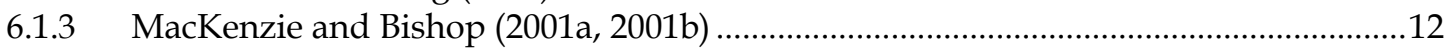

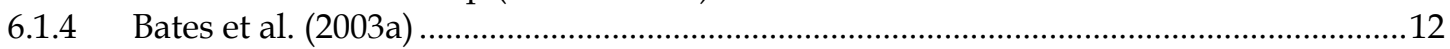

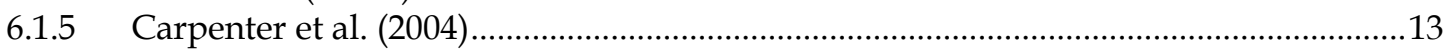

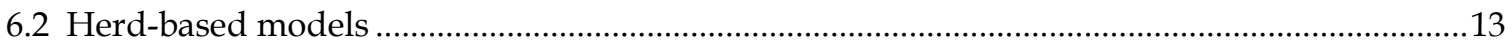

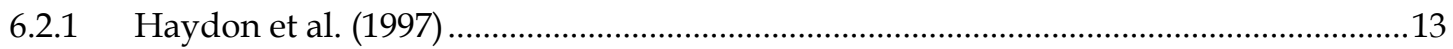

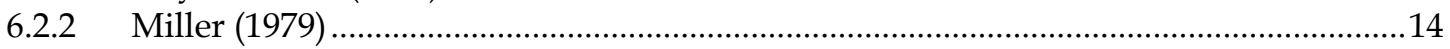

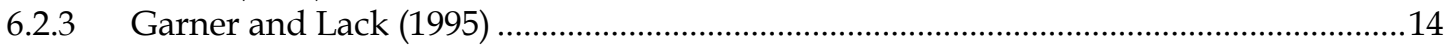

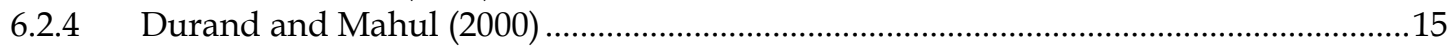

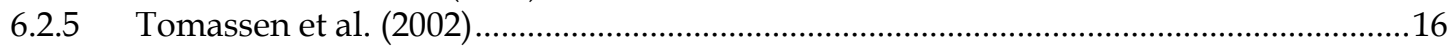

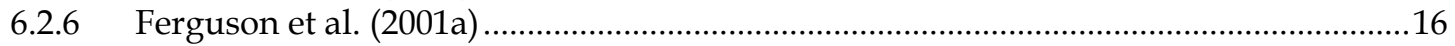

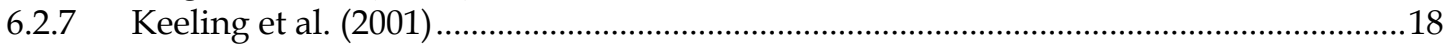

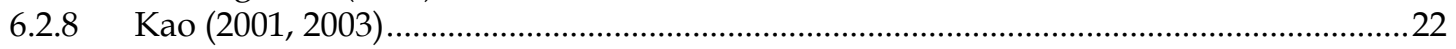

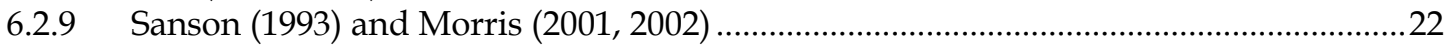

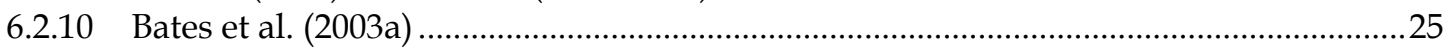

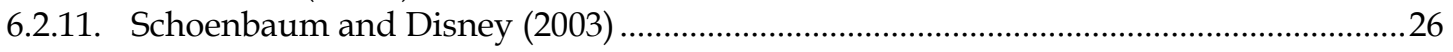

7.0 Feature-by-Feature Comparison of All Models ……..........................................................................28

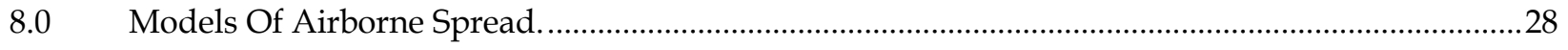

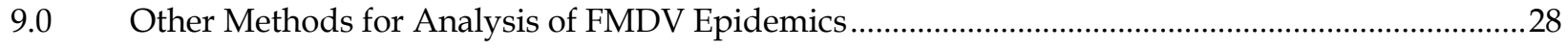

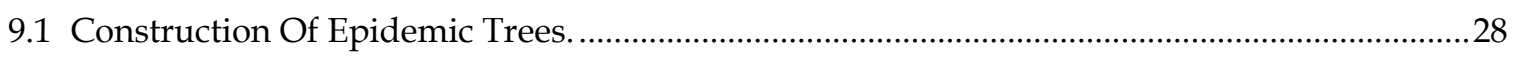

9.2 Visualizing The Dynamics Of The Epidemic. ……..........................................................................

9.3 Geographic Information Systems (GIS) And Correlation Analysis ............................................30

9.4 Methods For Optimization Applied to Control of FMD ................................................................30

$10.0 \quad$ Proposed Research and Development.............................................................................................. 


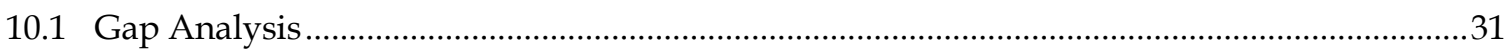

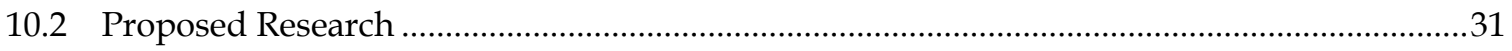

10.2.1 The Grand Challenge: Development of a Decision-Support System............................31

10.2.2 Basic Research to Support the Development of a Decision Support System................32

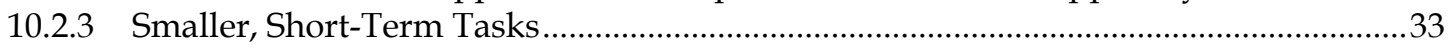

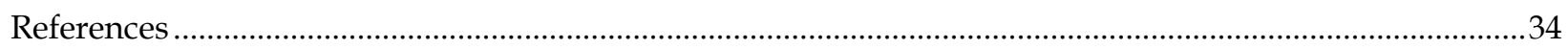




\section{I.0 Introduction}

The 2001 outbreaks of foot-and-mouth disease (FMD) in Europe (Ferguson et al. 2001a, 2001b; Bouma et al. 2003) and concern about the possibility of an intentional introduction of a devastating foreign animal disease triggered renewed interest in both theoretical and experimental research related to FMD. Theoretical models of disease transmission, which influenced the tactical decisions of the United Kingdom (UK) government during the epidemic (Taylor 2003), resulted in large numbers of uninfected animals being slaughtered. After the epidemic, the adopted control policies were sharply criticized (Kitching 2004;, Taylor 2003). Still, the role of computational modeling for analyzing the scope of the epidemic and devising control strategies was recognized as substantial and necessary. In its summary and recommendations, the Royal Society Committee on infectious diseases in livestock concluded

"Quantitative modelling is one of the essential tools both for developing strategies in preparation for an outbreak and for predicting and evaluating the effectiveness of control policies during an outbreak. A prerequisite is a central database incorporating improved data on farms, the location of animals, animal movements, and the characteristics of the diseases, together with arrangements to input disease control data in a timely and assured way during an outbreak. More work is required to refine the existing models and to strengthen their capacity to inform policy, which in turn requires full access by researchers to this database and to the data on previous outbreaks. (Royal Society 2002)"

The modeling approaches and assumptions have been re-evaluated in reviews by Taylor (2003), Kao (2003), Wilson et al. (2001), Sutmoller et al. (2003), Leforban and Gerbier (2002), Grubman and Baxt
(2004), and Green and Medley (2002). However, these authors reviewed only the UK models and some of the international models developed prior to the 2001 FMD epidemic.

In this paper we review all modeling efforts since 1990. Before summarizing the results of our study, we highlight three important points.

- First, epidemic models in general have limited predictive value. However, in contrast to human epidemics, which are difficult to assess because of the mobility of contemporary societies, models of livestock disease can be more reliable because livestock are managed in a relatively structured manner. Livestock locations can be determined, the management processes on each farm can be simulated and evaluated, and (with farmers' consent and the introduction of modern technologies), good estimates of contact data can be made. Furthermore, as an illustration of what is possible, in Denmark, all livestock locations and all individual animal movements (in excess of one million) are recorded. These locations and movements are prerequisites to the development of reliable models and decision support systems to be used in an epidemic and to train veterinary personnel between epidemics.

- Next, it is not a coincidence that much of the modeling of livestock epidemics has been performed for FMD. FMD is the most economically devastating livestock disease, and, from the modeler's point of view, it is representative of all nonarthropod-borne livestock diseases. Thus, a model of FMD can be adapted and parametrized for other livestock viral and bacterial diseases.

- Finally, the pragmatic value of epidemic models is measured in two ways: their capability to evaluate the effectiveness of 
control measures, and their capability to estimate the cost of an epidemic. Thus, epidemic models with control consist of a baseline epidemic model and superimposed models of control (Figure 2). While the simulation and evaluation of the methods of epidemic control is a relatively well defined and straightforward task, the baseline epidemic model has to account for numerous, still not fully understood, factors. The reliability of evaluations and predictions depends crucially on the reliability of the baseline epidemic model. For this reason we focus on the features of each method that might lead to unrealistic representations of an epidemic and therefore would skew the evaluation of control strategies.

\subsection{Goals}

The goal of this paper is to review the entire range of FMD modeling efforts undertaken since 1990.

We have three specific purposes:

1. To evaluate the relative strengths and weaknesses of each model and of the types of modeling approaches.

2. To highlight shortcomings that could be overcome through novel computational modeling efforts;

3. To identify new directions in quantitative research that would contribute to optimizing methods for livestock disease control.

\subsection{Summary of Results}

The modeling approaches included in this study can be categorized in three groups:

1. Intra-herd or on-the-farm epidemic models that estimate the scope and speed of disease transmission within a single herd or on a single farm.
2. Herd-based (or farm-based) FMD epidemic models that describe disease spread over large areas populated by many herds (or farms).

These models are either non-spatial or spatial.

3. Various quantitative approaches contributing to an understanding of FMD transmission.

Plume models for the estimation of airborne virus transmission are briefly mentioned but are not a part of this review.

The models can also be categorized in two general groups: "top-down" and "bottom-up" approaches.

Top-down approaches hypothesize macroscopic behavior and fit parameters of the constituents to demonstrate that behavior fits data. They use closed formulations in the form of systems of differential equations of discrete time-dependent equations with a relatively small number of parameters that are fitted to available data. The parameters often have a relatively abstract meaning and the values obtained after fitting do not have universal value that can be used repeatedly.

Bottom-up models obtain macroscopic behavior from knowledge of the constituents. They are usually probabilistic rule-based, detailed quantitative descriptions of the infection process, using a relatively large number of data-based parameters.

A comprehensive comparison of the models with regard to the incorporated detail is included in Section 7.

The results from our analysis lead to the following conclusions:

- All existing intra-herd models are too crude to supply reliable estimates for the speed of epidemic spread within a herd. Studies of the 
contact rates between animals in a herd do not exist. Intra-herd and on-the -farm models need to and can be refined to reflect the management practices on the farm. Models should differentiate farms that are managed and organize the production process differently, and for each type the scope of spread of simulated epidemics should be analyzed and compared. This can bring about changes in management practices that reduce disease transmission if there is an outbreak.

- Non-spatial farm-based models (i.e. those that assume random contacts and do not incorporate spatial factors such as the distances between farms and their geographic locations), such as those by Carpenter and Thieme (1979), Miller (1979), Haydon et al. (1996), and Ferguson et al. (2001), rely on averaged farm densities and dissemination rates. They ignore important epidemic transmission factors and thus represent a rather crude representation of an epidemic.

- In our opinion, bottom-up models are better fit to provide answers to the practical problems of strategic planning and cost/benefit analysis. Top-down models are better suited for postepidemic analyses.

- The top-down model by Keeling et al. (2001) is an elegant way to simplify the complexity of the epidemic process. It used detailed spatial and UK farm data and represented the probability of disease transmission between an infected and any other susceptible farm by using only three unknown "lumped" parameters (referred to as sheep and cattle transmissibility and cattle susceptibility). Our analysis concludes, however, that the model makes too many simplifying assumptions to be a realistic representation of the epidemic process. It is even less realistic for the type of farm sizes and management practices characteristic for the US.
- Spatially-uniform farm-based models that simulate artificial (synthetic) spatial farm distributions generated randomly like those of Garner and Lack (1996), Durand and Mahul (2000) and Schoenbaum and Disney (2003) ignore the usual spatial heterogeneity of farm distribution. Spatial farm aggregation is supposed to affect disease transmission within the aggregated farm groups and disease spread between groups in opposite ways, speeding up the first and slowing down the second. Thus the total effect of spatial heterogeneity is complex. Therefore, although such models have been heavily used to evaluate control strategies and perform cost/benefit analysis, the validity of their conclusions is doubtful.

- Bottom-up spatially-explicit models like this by Bates et al. (2003a) and the decision-support system EpiMan of Sanson and Morris (Sanson 1993, 1999; Morris et al. 2001, 2002) incorporate a multitude of factors defining disease transmission and use realistic spatial farm distribution data. The model of Schoenbaum and Disney falls in the same category if realistic spatial farm distributions are used. In our opinion, this is the type of modeling approach that should be used to assess disease transmission and evaluate control strategies.

- None of the models in their present form can meet the US needs for an epidemic modeling system that can be used for the purposes of disease control planning and prediction of disease spread. The most developed tool for these purposes currently is Epiman (Sanson 1993; Morris 2001). However, first, it is a commercial product that is not amenable to modifications that will be needed; next, it is unclear whether it is suitable for the US specifics that would require large-scale simulations. From the two existing US epidemic simulation models, the one by Bates 
et al. (2003) incorporates the most detail and realism.

- $\quad$ To meet US livestock epidemic modeling needs an effort to develop a fast-speed largescale model must be undertaken. It will take the least effort to develop a realistic system if the epidemic part of the model is based on an improved version of the model by Bates et al. 2003 that overcomes some of the deficiencies of this model (improved intra-herd model, inclusion of airborne transmission module, inclusion of the road network and known epidemic network structure) and is large-scale and computationally efficient (allowing highspeed computation for large-scale spatial dimensions and herd numbers). The model should be accompanied by an economic module, which could combine the features and expertise of the two US models (Bates et al. 2003 and Schoenbaum and Disney 2003).

- The development of the system will require non-trivial efforts combining large-scale computations expertise, collection and manipulation of various additional data and collaborative work of experts.

An outline of the system's desired features and additional, smaller-scale tasks that need to be addressed before or during the development of the system are presented in Section 10.

\subsection{Background Information on Epidemic Models.}

\section{I The General Architecture Of Epidemic Models.}

Because infectious disease is a manifestation of a host-parasite relationship in which the parasite, or agent, impairs the normal functioning of the host, one approach to epidemic modeling is to model the dynamics of the host and parasite populations. Such models exist (for example, Bouloux et al. 1998; Rosa et al. 2003), but from the modeling point of view, a detailed representation of the dynamics of both the host and the parasite complicates the problem considerably.

To reduce the modeled complexity of host-parasite dynamics, the usual approach to epidemic modeling is to subdivide the host population into classes, called states, such as susceptible (the individual is healthy, but prone to infection), latent (the individual is infected, but not yet infectious), subclinical (the individual is infectious, but not yet symptomatic), clinical (the individual is symptomatic and infectious), recovered and temporarily immune (the individual can return to the susceptible state after some time), and removed (the individual is dead). Thus, if initially there was no infection, the whole host population would be in the susceptible state. The latent, subclinical, and clinical are infected states. The subclinical and clinical are infectious states. Rules of transition from state to state are then defined.

Figure 1 represents the fundamental architecture of a general epidemic model, typically referred to as a state-transition model. As simple as the structure appears, the implementation of such models can be accomplished with many different approaches and levels of detail (briefly presented in the following subsections), thereby complicating efforts to compare different models.

The most influential parameter of any statetransition model is typically the rule that defines the transition from the susceptible to the infected state. It is widely accepted that this rule, which is known as infection rate in deterministic models and infection probability in stochastic ones, is positively correlated with the quantity of "infectious contacts." However, quantifying what constitutes a "contact," how infectious it is, how many contacts can occur, and on what occasions a contact implies occurrence of infection can also be done in different ways and in different levels of detail, as is seen in the models reviewed in the next sections. 


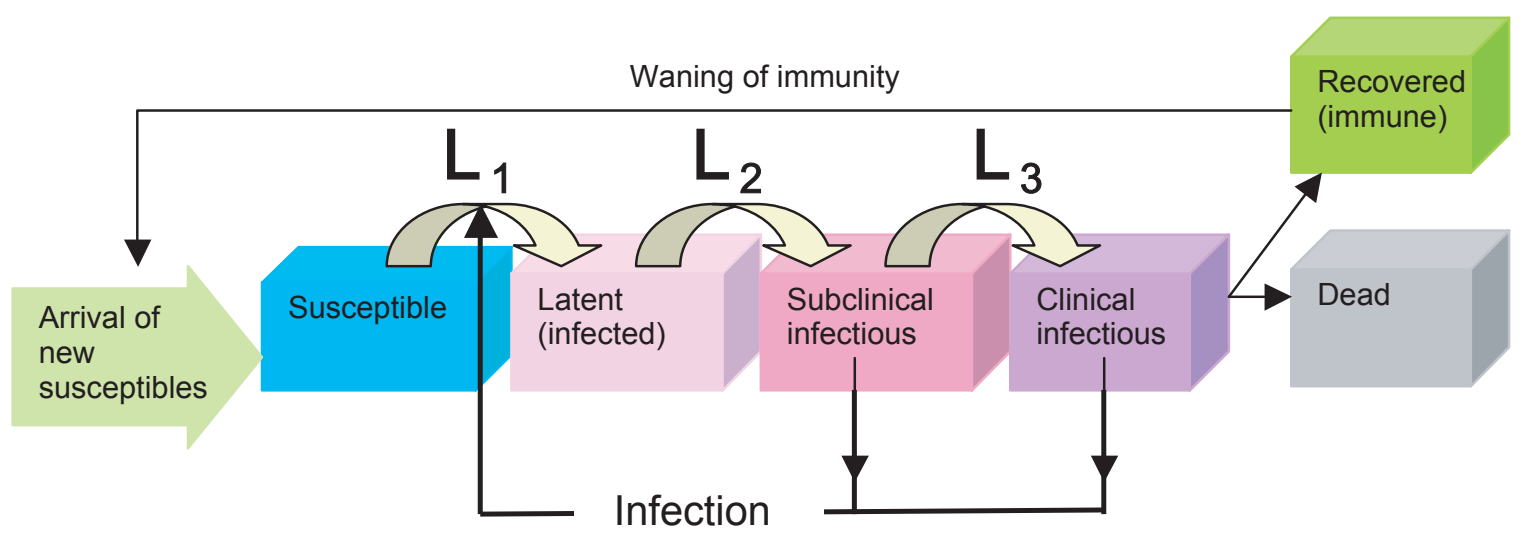

Figure I. Schematic representation of an epidemic model.

The rules describing the transition between any of the infected states are usually based on knowledge about the average time an individual spends in an infected class before passing to the next (duration period, e.g., incubation period, subclinical infectious period). In deterministic models, the transition rate is the inverse of the duration period, while stochastic models apply a procedure to choose a random transition time to the next state within the length of the duration period.

One weakness of basing transition rules only on knowledge about transition times is that the dependence of the transition rate on the quantity of virus that initiated the infection is ignored. Experimental evidence for some pathogens suggests that the incubation period is shorter if the amount of infectious particles ingested (the infectious dose) is larger and vice versa. However, because the exposed dose is currently difficult to quantify, models typically ignore it.

\subsection{The Bottom-Up and Top-Down Modeling Approaches}

The top-down approach in modeling is based on pre-set assumptions about the form of the components in the model. In epidemic models, this results in assuming certain formulas for the transmission rates, which involve a few lumped parameters (incorporating many factors) that are either found by fitting the model to data or varied in certain sets of values that are assumed to be realistic. Conclusions are then based on results from the model obeying these assumptions and are as truthful as the model's assumptions. These models are usually deterministic in nature.

The bottom-up approach is a computational approach that attempts to mimic the process by representing its constituents as virtual objects with parameters (attributes) derived from "real data." Thus, bottom-up models may incorporate topdown models of the constituents. These models split the epidemic process into many components (for example, describing the assumed details of infection transmission), thus requiring the use of many "real-data" parameters. Because epidemiological data are fuzzy, these models are necessarily stochastic, based on Monte Carlo simulations, the parameters being defined by using certain probability distribution functions. Conclusions are based on the averaged simulation results, and validation is possible only in comparison with previous epidemic results. Even if the input data are reliable, the validity of the conclusions depends on the assumptions for the probability distributions, the way in which the computations are performed, and the number of simulations carried out. 
Complex computational models represent combinations of top-down and bottom-up approaches.

\subsection{Types Of Epidemic Models With Regard To Spatial Detail}

State-transition epidemic models can be continuous or discrete. The continuous models usually represent deterministic differential equations. Some of these can incorporate spatial variables (they model animal movement in space as a diffusion process), but in most cases the deterministic continuous models are non-spatial. The discrete models can be deterministic or stochastic, and each of these can be spatial or nonspatial.

\subsection{Non-spatial Models}

Non-spatial models deal with the total numbers of individuals in the various disease classes ignoring their actual spatial distribution. The best-known epidemic models are deterministic continuous models and are typically based on a system of ordinary differential equations (ODEs). This modeling class is commonly referred to as a SIR (Susceptible-Infectious-Recovered) model. ODE SIR models use constant transition rates between the infected and recovered classes. For example, the equation describing infectious individuals assumes that they increase by recruiting some latent individuals and losing some individuals to the recovered class. An example of a transition equation is

$$
d l / d t=(1 / \tau) L-(1 / \sigma) R
$$

where the transition rates are the inverses of the average times individuals stay in the exposed and infected classes. The transition rate between the susceptible and exposed class is proportional to the density of the infected class, with a constant of proportionality. The corresponding equation is

$$
d S / d t=-k[I /(N)] . S,
$$

where $\mathrm{N}$ is the total population. Similar equations are derived for the remaining transitions between the other classes. Thus, in deterministic ODE models, all animals in a given state have the same probability of passing into the next state and the transition rules contain rate constants, which are fitted to existing data. An example is the smallpox model of Gani and Leach (2001).

A different approach uses stochastic statetransition (SST) SIR models, which are usually discrete models. SST models incorporate expected variation into the transition rules. These rules take the form of transition probabilities. SST models also model individuals (animals, herds) and their epidemic states, as opposed to populations in deterministic models. Transition times between the states, as well as the number of infected animals, are calculated for each animal from predefined probability distribution functions.

The time-since-infection structured (TSI) models are a form of deterministic continuous models. However, TSI models are constructed in a way that allows variability in the transition times. They include partial differential equations to track the time since entering the class as an independent variable. For example, at time $t$, the infectious class is described by $I(t, \tau)$ - the density distribution function of the individuals infected $\tau$ units of time ago. This representation of the disease stages allows for the inclusion of probability distributions in a deterministic model. For example, if $\lambda(\tau)$ is the probability that a person who has been in the infectious class since time $\tau$, will recover, then the transition rate of transfer from the infectious to the recovered class is

$$
\int \lambda(\tau) I(t, \tau) d \tau
$$

and the integration is performed from zero to the maximum possible time of staying in the infectious class. Time-since-infection structured models are 
not often used by the epidemic modeling community because of their mathematical complexity, but they are steadily gaining popularity (Kaplan et al. 2002, Martcheva and Castillo-Chavez 2003; Feng et al. 2002).

Because differential equation models work with continuous functions while epidemic numbers are integers, discrete versions of the same models are sometimes used. These models are referred to as discrete deterministic state-transition models.

\subsubsection{Spatial Models}

All of the models presented above do not include a spatial dimension, but spatial parameters play an important role in disease transmission. For example, the effectiveness of an infectious "contact" to transmit infection is known to depend on the distance between the contacts, the duration of the contact, and many other properties of the pathogen, host, and environment. Individual susceptible and infected units are rarely distributed uniformly in space. Most often they are aggregated in localized groups, and this becomes an important factor in the speed and proportions of an epidemic. But even if there were no aggregation, individuals are not likely to mix continuously and uniformly: an assumption all non-spatial models make in defining their infection rates.

Spatial state-transition models of human and animal populations have a common problem: the uncertainties associated with movement. A spatial model must define how individuals move in space and how infection is transmitted between individuals that are located at various points in space. This can be accomplished with different degrees of detail.

When exact spatial information is not available, the usual procedure is to generate artificial spatial uniformly-random distributions of individuals (farms, animals) and use a so-called "spatial kernel" to define the probability of infection transmission as a function of the distance between infectious and susceptible individuals. In such a model, the probability of an individual passing from one disease state to another depends, among other factors, on the distance to other individuals, but not on the specific spatial locations or other significant spatial factors. For our purposes, we shall refer to such spatial-state-transition models as spatially-uniform models.

Spatially-uniform models do not consider important spatial features such as aggregation of individuals. Aggregation of individuals contributes to re-infection and prolonging the infection and to faster disease transmission within the group because of the higher density of individuals in the aggregated groups. At the same time, the existence of spatially aggregated separate groups slows down the infection process because of the lower probability of infection transmission between spatially remote groups. The combination of these effects can either prolong or shorten the epidemic. Thus, the spatial patterns of grouped individuals is important in determining the outcome of the epidemic.

When the epidemic unit is a farm or herd, the geographic infrastructure of the region where the farm is located is important. Farms usually exist in aggregated groups, and the above arguments for the complex role of aggregation in disease transmission hold. The physical distance between two farms is an important factor in disease transmission (relatively close farms are more likely to have "contact", i.e. exchange viruses). The existence of a road connecting two farms may be of more importance when the farms are relatively far from one another because the road increases the possibility of indirect transmission. Thus, in addition to the distance between farms, spatial models can contain exact spatial locations and 
incorporate urban infrastructure and other geospatial information. Such models belong to the class of spatially explicit models.

Spatial models are especially suitable for modeling and simulation of epidemic control strategies.

\subsection{Types of Models with Regard to Purpose}

Analytical models are used to gain insight into existing epidemic data and to explain observed phenomena and relations. These are usually "simple" (in form) models that are analyzed theoretically (not always an easy task!). Analytical models produce general conclusions of cognitive value.

Predictive models are designed to project the scope and speed of the epidemic. Strategic evaluative models mimic an epidemic and are used to compare the effect of different control strategies. Economic evaluative models are based on evaluative models with control, but they have additional tools to estimate the cost of an epidemic and the economic impact of applied strategies. Decision-support systems use simulation models and economic models to assist managers in handling complex epidemic situations.

\subsection{Epidemic Models for Evaluating Control Policies}

Once a baseline epidemic model is constructed, various methods of intervention can be evaluated by removing or altering the immunity status of individuals. Figure 2 illustrates some of the control measures that can be simulated in a livestock disease model.

Important variables here are the duration of the delay in implementing controls, as well as variables measuring the effectiveness of control performance (for example, vaccine efficacy, percentage of infectious animals slaughtered or presumably susceptible animals vaccinated per day, because of constraints). By simulating the

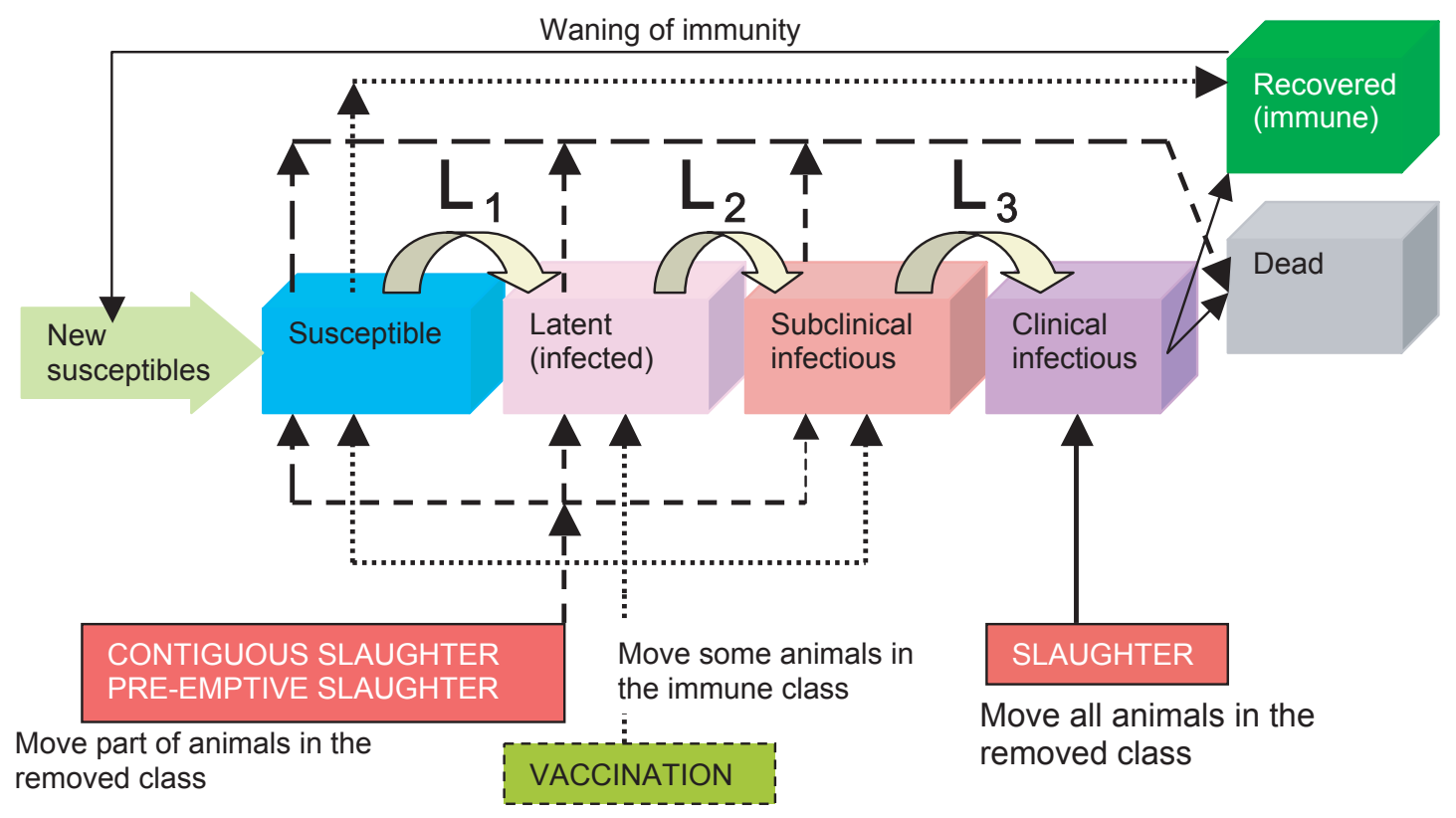

Figure 2. Schematic representation of the action of control strategies superimposed on a baseline epidemic model. 
application of the controls at various degrees and in various combinations, it is possible to estimate the corresponding gains and losses and select the most effective controls (according to a given criterion). For example, the losses can be expressed in terms of the number of healthy animals slaughtered, animals infected, expenses for slowing down the epidemic by vaccination, and the total cost of the epidemic. The final criterion measuring control success is a reduction in the total cost of the epidemic. Thus, simulations with the model can lead to a ranking of control measures with regard to epidemic costinformation that can be useful for decision-makers. Also, control strategies can be defined by parameters such as the radius of the ring of preemptive slaughter or of the vaccination ring around an infected farm. By varying these parameters, it is possible to reduce the costs by determining the optimal slaughter and vaccination ring radii combination. In this way, the solution of the optimization problem would point to the most adequate strategy for the specific region, instead of the "blind" comparison of a certain number of predefined control strategies.

It is important to note that before evaluating control measures, the basic epidemic model requires accurate data - data that, as a rule, are not available. Usually it is assumed that data driving the model belong to some set of values. For example, the number of indirect contacts expected by a specific individual is presumed to be in certain bounds and governed by certain probability density functions. To some degree, this assumption already predefines the optimal control measures. If the epidemic model data are assumed to belong to a different set of values, another optimal strategy may be obtained.

In addition, the optimal control measures might depend on the scope of the epidemic. If the epidemic is intentionally started, it might have many simultaneous and spatially distant sources. Such an epidemic may require different control measures than a smaller, unique-source epidemic.

A given control measure might be robust with respect to performance, meaning that it ranks best under all data assumptions. Determining such a control via modeling is well worth the effort.

\subsubsection{Economic (Cost/Benefit Evaluation) Models}

Without economic analysis, control measures can be compared only on the basis of how fast an epidemic ends or how many individuals are affected. Economic models take into consideration the consequences of an epidemic and its control along the chain of impacted industries, exports, and human lives. One of the first simulation tools for economic analyses of an FMD outbreak was proposed by Carpenter and Thieme (1980).

\subsection{Modeling Disease Transmission Among Livestock Populations}

As livestock are typically managed in spatially restricted locations (herds), epidemics can progress rapidly within the herd. Some diseases such as FMD spread so fast and can be so difficult to control within a herd that it is convenient to regard the epidemic as developing in a population of herds and not individual animals. Therefore, for most livestock disease epidemic modeling, a whole herd is considered to be infected if a single animal in it is infected, but the level of infectiousness may vary depending on the model. All of the FMD epidemic models reviewed here regard herds as the primary modeling unit.

\section{I Factors for Disease Transmission in Livestock}

The factors governing the incidence, spread, and control of a non-specific livestock disease can be grouped in three categories. 
1. Individual animal disease factors, such as involved species, their specific in-host pathogen dynamics (including specific pathogen strain variability, formation of immunity, vaccine protection, incubation period, subclinical period, infectious period, average quantity of shed pathogen, dependence of peak infectiousness and length of infectious period on the dose of pathogen, ease of detection of clinical signs),; the agespecificity of the pathogen dynamics;

\section{On-the-farm epidemic factors}

a) direct transmission factors: number of animals of different species on the farm; rates of pathogen transmission between individual animals of the same and different species (pathogen range in case of airborne transmission, quantity of present pathogen in excreted fluids: saliva, urine, feces, milk); average number of susceptible contacts of an infected animal;

b) indirect transmission factors: management of farm that contributes to contamination of common areas, quantity of human-animal contacts, viability of pathogen in the soil, air, or water.

\section{Between-farms transmission factors}

a) Frequency and magnitude of direct contacts between farms (expressed by exchange of animals between farms) and between farms and collection points (sale yards, distribution centers);

b) Frequency of indirect contacts between farms (incoming and outgoing vehicles, personnel visiting between farms), visits and deliveries from outer sources, road and other transportation network connecting farms, weather factors contributing to airborne transmission, geographical factors amplifying or reducing speed of airborne transmission.

The different types of factors are inter-related. For example, the size of a farm determines the amount of movement in and out of the farm and, therefore, the direct and indirect between - farms contacts.

\subsection{Specifics of Foot and Mouth Disease Spread and Their Significance for Epidemic Modeling}

A superb review on the pathogenesis and clinical development of FMD in the various host species was published by Alexandersen et al. (2003a). Below we give a short summary of the specifics of foot-and-mouth disease virus (FMDV) infection along the lines of the previous section.

The incubation periods for FMD are known to vary by species. The incubation period depends on the dose of received virus as well as on the type of infection. Although incubation periods have been reported to be 5-8, 3-5, and 5-13 days for pigs, cattle and sheep, respectively (Bates et al. 2003), these limits can be expanded to be somewhere between 2 and 14 days for all species. The mean subclinical periods were reported to be $4.3,2.2$, and 2.6 days (Bates et al. 2003). The total infectious period is more difficult to quantify because it can have wide bounds. The variable lengths of these periods reflect the dependence on the initial dose of received virus as well as the response of the hosts' immune system.

The immune response - virus intra-host dynamics determines the peak and average virus quantities a host excretes. Hughes et al. (2002) demonstrated in experiments with sheep that the peak viral excretion decreases with increasing infection dose, at least for dose amounts in certain limits. Also, the incubation period and the infectious period decreased with increasing dose. Similar doseresponse relationships in pigs and cattle has not 
been reported but research on virus-immune response dynamics is underway (Alexanderson and Donaldson 2002;, Alexandersen et al., 2002a, 2002b, 2003b).

The species heterogeneities with respect to disease transmission are a result of an evolutionary process of adaptation of the virus and hosts that guaranties the persistence of the virus. Thus, a model that disregards the heterogeneities of the different species present in a modeled region might substantially misrepresent the dynamics of an epidemic

The FMDV is an RNA virus that exhibits quasispecies dynamics. This means that viral populations even in the same host can be genetically heterogeneous. Even the progeny of a single genome are genetically heterogeneous (Domingo et al. 2003). This has been demonstrated both in vitro and in vivo (Domingo et al. 2003). The significance of these observations is that FMDV could mutate very rapidly (similarly to influenza viruses). However, it is generally not considered to be a rapidly changing virus (Bates 2003). To protect animals from each viral serotype requires a vaccine strain homologous to the outbreak strain because vaccines derived from one serotype do not cross-protect against the other serotypes. Some FMDV strains have been shown to be more genetically variable, causing the efficacy of the vaccines against these strains to be lower (Wilson et al. 2001). The genetic heterogeneity of viral populations inside individual hosts is the prerequisite for the formation of poor host immunity and the existence of persistent infections and carrier states (Sutmoller et al. 2003). Epidemics caused by the different serotypes can differ substantially in their speed and means of spread. The difference has yet to be understood. Thus, identifying the strain of the causative agent and quantifying its immunologic and epidemiologic features is a very important factor for predicting and controlling the epidemic.

FMDV infects several host species: cattle, sheep, goats, pigs, and some wildlife species such as deer. There are two main routes of infection which differ in their quantitative characteristics in the various species: the oral route (through food contamination) and the inhalation route (through inhaled airborne particles). The virus infects pigs more easily by the oral route and is secreted in larger quantities through exhalation in pigs than in other species. One study (Alexandersen and Donaldson 2002) suggests that pigs need about 60 times more virus to be infected by inhalation but secrete 60 times more virus by exhalation than cattle and sheep. Inversely, FMDV infects cattle more easily (needs less virus particles) via the inhalation route than via the digestive route but this species secretes large quantities of virus in body fluids that contaminate the surroundings. Sheep need similar doses to become infected and exhale similar concentrations of virus as cattle, but are smaller and shed less virus in total. However, sheep typically display only mild clinical signs and can go undetected for long periods of time.

The existence of a relatively long (up to 48 hours) subclinical period is a significant feature of FMDV. It contributes significantly to the spread of FMDV both by the direct animal-to animal contact and indirect mechanical transfer by vehicles, human contact, and other indirect means.

The number of indirect contacts depends on the farm type (beef, dairy, pig fattening), on the species present (cattle are more often in contact with humans than pigs, for example), on the farm size, on the density of farms in a region, and even on the farm fragmentation (number of parcels in a farm). FMDV has been shown to remain viable for months in the soil, especially in colder seasons. 
The role of roads and geographic infrastructure in the transmission of infection has been hypothesized by several authors (Keeling et al. 2001, Rivas et al. 2003). Contemporary farm managing practices contribute to the establishment of long-range indirect contacts between farms as vehicles and agricultural machinery are exchanged between far away premises. Moving animals at very long distances is a frequent practice as well, establishing long-range direct contacts between farms.

\section{0 Review of Foot-and-Mouth Disease Models}

\section{I. Intra-Herd (On-the-Farm) Models}

It is generally accepted that the infectiousness of an infected farm increases with time as more animals become infectious. One approach to account for this phenomenon is to vary the infectiousness of an individual farm, based on the time since it was infected. A few modeling papers include simple intra-herd models within a broader epidemic model to vary the level of infectiousness of a herd. Some works are specifically dedicated to intra-herd modeling. We summarize these efforts below.

\section{I.I Sanson (1993)}

Sanson's model (Sanson 1993) is described in detail below (EpiMan model). It is a detailed model attempting to represent the amount of virus produced by a herd over time. However, the central assumption in the model is the cohortbased transmission of infection, which is very artificial.

\section{I.2 Hutber and Kitching (1996)}

Hutber and Kitching (1996) proposed a stochastic state-transition model of FMD infection in a herd vaccinated post-infection. This model is unique as it uses immunological data from Saudi Arabia outbreaks to simulate the level of antibody titres in individual animals. The authors call the model "vector-transition"; however, it can also be described as a discrete time-since infection model as the state of each animal actually depends on the time since infection occurred. The model results are not well described because the paper is mainly an explanation of the technique developed and used.

\section{I.3 MacKenzie and Bishop (200 Ia, 200 Ib)}

MacKenzie and Bishop (2001a, 2001b) proposed a stochastic simulation model of an infectious disease on a pig farm with only two disease stages: susceptible and infectious. The implementation of the model is a discrete event simulation with two components: event type and time until next event. The event types are either an animal becoming sick or recovering. This simple simulation model is then applied on a hypothetical pig farm. The pig population is age-structured and there is a welldefined schedule of managing the pig farm. The model is generalized to include genetic variability with respect to disease susceptibility and is used to analyze the consequences of selecting animals for resistance to the disease. An interesting result from the model is that the probability of an epidemic is dependent on the index case type (i.e., whether it is a nursery pig or a gestating sow) as well as on the organization of the farm production process.

\section{I.4 Bates et al. (2003a)}

Bates et al. (2003a) formulated a method based on the Reed-Frost model (Noordhuizen et al., 2001) to calculate the approximate quantity of infected animals $I_{t}$ after time $t$. This is a discrete deterministic SLI model, which represents a discrete version of a time-since-infection model.

$$
I_{t}=\sum_{i=0}^{t-\lambda} L_{i}
$$

where 


$$
L_{t+1}=S_{t}\left(1-q_{t}^{\prime}\right), \quad S_{t}=N-\sum_{i=0}^{t} L_{i}
$$

and

$$
I_{t}=0
$$

when

$$
t<\lambda+1
$$

In the equation above $q=1-k / N, \lambda$ is the latent period, $k$ is the number of contacts and $N$ is the total herd size. The value of $k$ was estimated from the half-time of infection of the herd. The model was used as a part of their larger herd-based model (Bates et al. 2003a). This model, however, assumes that an infectious animal remains so forever; thus, the number of infections is overestimated.

This model can be amended if the average infectious period $D$ is taken into consideration as follows:

$$
I_{t}=\sum_{i=t-\lambda-D}^{t-\lambda} L_{i}
$$

where

$$
L_{t+1}=S_{t}\left(1-q_{t}^{\prime}\right), S_{t}=N-\sum_{i=0}^{t} L_{i}
$$

and

$$
I_{t}=0
$$

when

$$
t<\lambda+D+1
$$

\section{I.5 Carpenter et al. (2004)}

Carpenter et al. (2004) formulated a discrete deterministic model of intra-herd epidemic of the
SLIR type which, with a slight transformation, is brought in the above form. The goal of the model is to estimate the time for which the number of infected animals will reach a certain percentage that would guarantee detection of some of the clinically ill animals.

Another form of the above equations, involving only the infectious and the susceptible class is:

$$
\begin{aligned}
& I_{t}=\sum_{j=0}^{D} S_{t-\lambda-j}\left(1-q_{t-\lambda-j}^{\prime}\right), \\
& S_{t}=N-\sum_{i=0}^{t} S_{i}\left(1-q_{i}^{\prime}\right), \\
& I_{\lambda}=1, I_{s}=0 \text { for } s<\lambda .
\end{aligned}
$$

\subsection{Herd-based models}

Models striving to represent a global foot-andmouth epidemic recognize the convenience of regarding the herd as a model individual. The rationale is that FMD is so infectious that the presence of a unique infected animal necessitates the immediate removal of the whole herd. A concise representation of the types of the reviewed models is shown on Figure 3

\subsection{Haydon et al. (1997)}

Haydon et al. (1997) developed a simple discrete deterministic state transition model with delays to evaluate the time-dependent transmission rate (a lumped direct and indirect transmission value) from data of the 1967-68 UK epidemic. It is worthwhile noting the combination of simplicity and practicality of this model for analyzing epidemic data. 


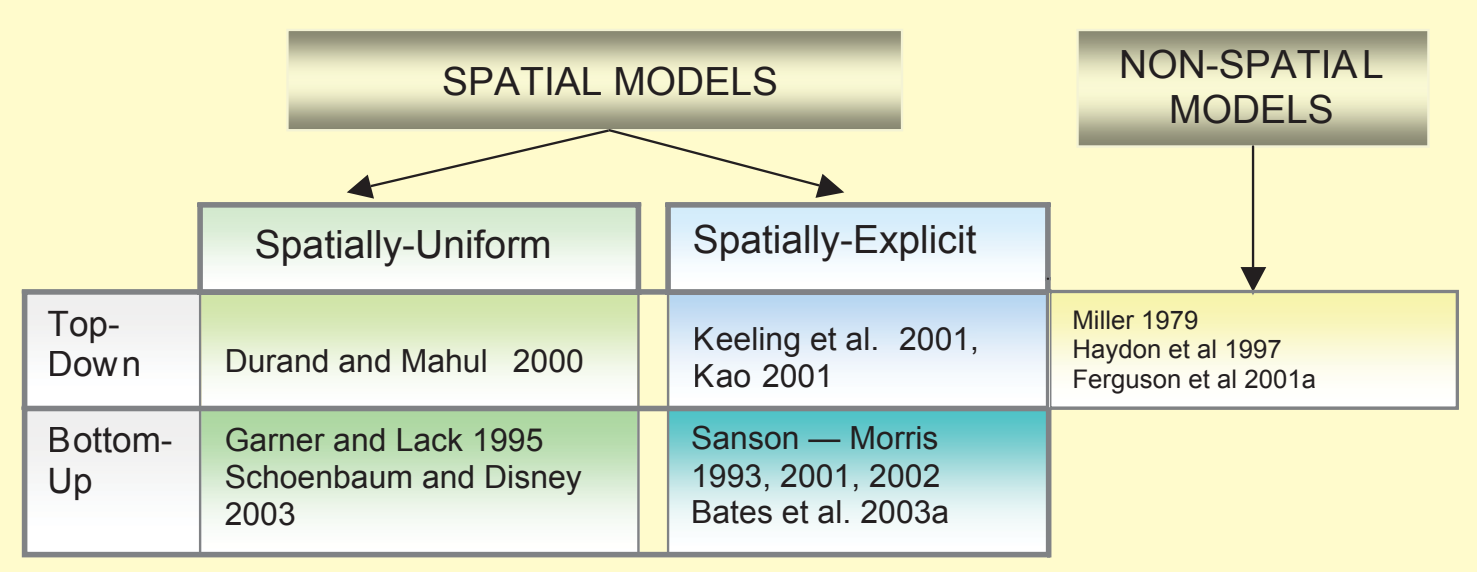

Figure 3. Types of the reviewed herd-based models

\subsubsection{Miller (1979)}

Miller's model (Miller 1979) is a simple deterministic state-transition model. The time unit is 1 week and there is no pre-clinical infectious state. The probability of transition of a herd from the susceptible to the infected state is $1-\mathrm{e}^{-D R}$. I, where DR is the so called dissemination rate constant and I is the number of infectious herds in the previous week $\mathrm{e}^{-\mathrm{DR}}$ is the probability of a susceptible herd to not become infected by any given infected herd. The constant DR is derived from the 1967-68 UK epidemic. This model is remarkable with the feature of first introducing the notion of DR. It invoked several follow-up models, as discussed in later sections.

\section{Critical remarks.}

All herds are considered equal and the model is not spatial. The model is used to estimate the scope of a US epidemic in case it developed with a similar DR as the 1967 UK epidemic. It is not clear how long an infected herd remain infectious and how long the immune period is. The lack of spatial considerations and the assumption that the DR is constant, are serious drawbacks of the model.

\subsubsection{Garner and Lack (1995)}

Garner and Lack's model (Garner and Lack 1995) is a stochastic spatially-uniform state-transition model. It introduced a number of new features in the realm of FMD modeling by recognizing the significance of herd size and herd density. The model is applied for three regions with different sizes and densities of herds corresponding to three Australian regions. The simulation starts with three different foci of infection and develops in circular regions around them. The transition probabilities are in the form of dissemination rates as in Miller (1979). However, because the model is stochastic, the dissemination rate is defined as the expected number of herds coming into contact with an infected herd. Each day the infected farms infect DR of randomly selected susceptible farms. The authors acknowledge the lack of realism in using a constant dissemination rate (as in Miller 1979) and attempt to mend this by changing the dissemination rate over the duration of the epidemic.

There are some elements of species dependency (for example, pigs are not vaccinated) although all transmission parameters do not differentiate between species. The model uses transition times between the states (latent period, infectious period and immune period), which are generated 
randomly from uniformly distributed values in predefined intervals.

The authors used the model to simulate four control strategies: a) slaughter of infected herds only, b) slaughter of infected herds and a certain percentage of the dangerous contacts (these are the infected but still incubating herds); c) slaughter of infected herds plus vaccination of herds within a ring of radius $10 \mathrm{~km}$ from an infected herd after 5 herds have been infected; $d$ ) slaughter of infected herds plus vaccination of herds in a ring with radius $10 \mathrm{~km}$ from an infected herd after 50 herds have been infected (late vaccination). Delays in the implementation of controls also were simulated.

The model also is the first with an economic component which calculates the cost of lost income and employment to the food-processing industry, the costs for containment of the epidemic, the direct livestock losses, and the costs for compensation payments. The indirect cost related to loss of trade was not included.

The results of the evaluation of the different strategies showed that strategy b) had the least negative impact in two of the three geographic areas considered. In one of the simulated areas the percentage of detectable infected herds and contacts was lower than in the others and strategies b) and c) were comparable.

\section{Critical remarks.}

It is not clear how the values of DR are selected. The only explanation supplied by the authors is that "regional values used for DR ... were estimated for each region taking into account the relative mix of enterprises, climatic factors, stock densities, .." (Garner and Lack 1995). The large modeling time unit (1 week) affects the precision of the model and forces large delays in application of the controls to be considered. The long time unit also makes the inclusion of a subclinical stage impossible as this typically occurs in the 2-3 day timeframe. A model with a shorter time unit and a subclinical stage might result in a very different dynamics of the epidemic and the comparison of strategies and economic loss may turn out to be different, especially for the case where strategies were comparably successful. A more accurate model might result in different outcomes when comparing strategies involving slaughter and vaccination in different proportions. Further critiques for this model can be found in the critical assessment of the Schoenbaum and Disney (2003) model.

\subsubsection{Durand and Mahul (2000)}

Durand and Mahul (2000) developed a

combination of a discrete deterministic model and a stochastic spatially-uniform model similar to Garner and Lack's. They used half a week as a time unit to represent dynamics of herd infection before diagnosis. The model has some features of intraherd as well as inter-herd disease dynamics. This model has the following epidemic states:

susceptible, exposed, latent, infectious but not diagnosed (which can be assumed equivalent to pre-clinical), diagnosed, immune, removed. Herd density data from two French provinces were used in the stochastic simulation model to generate the tentative dissemination rate (which has the same meaning as presented in Garner and Lack 1995). Having simulated the dissemination rate, this one and fixed transition times were used to evaluate the effect of three control strategies: slaughter, slaughter and dangerous contacts and slaughter and emergency vaccination by running the discrete deterministic model. A subsequent paper (Mahul and Durand 2000) was devoted to the calculation of the economic costs of the control strategies.

\section{Critical remarks.}

As the model is not spatially - explicit, the quantity of dangerous contacts was estimated as a constant fraction of all infected premises. All the model's 
parameters (mainly representing the transition rates between infectious states) are fixed at some predetermined values whose choice was not convincingly defended. It is unclear why the authors did not use their stochastic simulator to model the application of strategies explicitly but rather used a deterministic system to complement the stochastic simulation.

\subsubsection{Tomassen et al. (2002)}

Tomassen et al. (2002) used a previously developed deterministic model for swine fever epidemics and adapted it for foot-and-mouth disease by adding an airborne transmission model. The epidemic model was described very briefly and is difficult to assess. The epidemic model was then connected to an economic model to estimate direct costs and export losses. The costs were compared for several scenarios of control strategies using a decision tree software (Data 3.5 by TreeAge Software, Inc.) simulated for seven Dutch regions with different pig farm densities.

This work is remarkable with the observation of a relation between farm density and optimal strategy: ring vaccination is reported to be optimal for relatively densely populated areas, while ring culling is optimal for sparsely populated areas.

\section{Critical remarks.}

The model is too specific. It considers only one animal species (pigs) because it was adapted from a model specifically describing the pig rearing industry.

\subsubsection{Ferguson et al. (200 Ia)}

The model of Ferguson et al. (2001a) is a deterministic model representing a system of ordinary differential equations and using averaged information about the distance between farms. It was created with the goal to analyze data in the midst of the 2001 epidemic. It was published only 3 months after the start of the epidemic and was a fast attempt to derive some quantities from the data like the effective neighborhood size, the contact kernel and the basic reproductive number $\mathrm{R}_{0}$.

The basic assumption in this paper is that the infectiousness of a farm (i.e. how many farms on average are infected by an average infectious farm) can be represented as the sum $f(r)$ of the probabilities of long-range infection $p k(r) / \mathrm{N}$ and short-range infection, (1- $p) g(r)$. The long-range infection of a susceptible farm depends on the average density of farms $k(r)$ located at distance $r$. The short-range infection, termed local spread, is assumed to occur because of contacts between farms that are in the vicinity of the infected farm and depends on the distances between the infectious farm and the susceptible farms. The contact kernel $g(r)$ measures the infectiousness of an infectious farm with respect to a susceptible farm that is at distance $r$ units away. In this paper, the contact kernel is applied to short-range contacts and the dependence on distance $r$ is expressed in the form $\exp \left(-\alpha r^{\beta}\right)$. This form was chosen to fit epidemic data derived from infectious contacts data supplied by the UK Department of Environment, Food and Rural Affairs (DEFRA) during the 2001 epidemic. By fitting the function $f(r)$ to the available at the time data for the number and location of newly infected farms, the authors obtain values for the probability $p$ and the parameters $\alpha$ and $\beta$.

The authors also calculate the so called effective neighborhood size. Roughly, this is the minimum number of neighborhood farms that guarantees that at least one of them will become infected.

The most interesting feature of the paper is that it introduces a network structure as an important factor for infection transmission 
Ferguson et al. (2001a) proposed a model consisting of ordinary differential equations that were derived from a network structure. The epidemic classes in this model consist of susceptible, exposed, infected, removed, as well as of pairs of the type susceptible-exposed, susceptible-susceptible, exposed-infectious, etc. The infected class is in fact a set of several infected classes representing different stages of infectiousness and diagnosis. The model is not fully described, only its logic is outlined. The network-based logic of the model was borrowed from models of sexually-transmitted diseases (Keeling 1999, Kretzschmar 1996) which occur in a very natural way in a network of persons with specific preferences. The pairs represented the transmission of infection through the network at the short-range level, while the long-range contacts were modeled by using mass-action term representation reflecting a well mixed population of farms. The model was fitted to epidemic data and then used to predict the magnitude of the epidemic and the impact of control strategies such as pre-emptive slaughter by way of ring culling and ring vaccination.

\section{Critical remarks.}

The model and all considerations are highly averaged, ignoring the role of heterogeneity (neither the size of farms, nor the species constitution were taken in consideration). For example, the contact kernel of a given infectious farm is based on the average radial densities of susceptible farms from any infected farm. The averaging approach is necessitated by the modeling tool used - a deterministic ordinary differential equations system. All of the rates used in the model are constants; the only exception being the long-range probability which changes after the date at which the movement ban was introduced. Thus, the transmission rates between the infected states (incubation rate, etc.) are not variable. Even if it is assumed that the model is a correct representation of the epidemic dynamics, any estimate obtained with such a model would be quite crude.

The long-range versus short-range distinction of the probability of infection reasoning comes from statistical physics. The probability of infection is likened to the potential of a source which acts at short distances with a force inverse to the distance and at long distances with some baseline probability. The same reasoning was also used by Gerbier et al. (2002) who used stochastic point processes as a modeling approach. In our opinion, a mechanistic association between the infectiousness of a farm and the potential of a source is not well founded. The assumption that long-range transmission is uniformly probable is not realistic. Long -range transmission (at distances greater than $10 \mathrm{~km}$, the maximum range considered for airborne transmission) is affected predominantly by direct contact, mainly by movements of animals among farms and saleyards. Thus, long-range transmission is not of chaotic nature but is rather the consequence of well-defined and traceable contacts that can be represented in a network structure.

It is logical to expect that the infectiousness of a farm decreases with distance to a susceptible farm because of the loss of the amount of infectious particles with distance traveled. Ferguson et al. assume that the decay is expressed as a negative exponential of the distance to some power $\left(\exp \left(-\alpha \delta^{\beta}\right)\right)$. Other authors (Tinline 1972; Gerbier et al. 2002) used a different type of distance kernel, namely a negative power of the distance $\left(\rho \delta^{-\gamma}\right)$. The basic difference between these forms is the faster decay of the first one. Which kernel is more realistic is not known. In fact, in their second paper, the same authors abandoned the idea of predefining the form of the distance kernel. 
Results from simulations representing ring culling and vaccination with different radii were reported in this paper. However, as the model is not spatial, the simulation of rings at different distances was probably done by manipulating the averaged radial density of susceptible farms away from an average infected farm. It is not clear how reliable any predictions that require explicit spatial information are by using such an approach.

In conclusion, many of the model's key features are over-simplified and therefore any results from fitting it to data are suspect.

Ferguson and collaborators published a second paper in October 2001 (Ferguson et al. 2001b) in which a more thorough analysis of the epidemic data was undertaken. The so called spatial hazard model was created and used to estimate the farms' infectiousness $(\alpha)$, susceptibility $(\tau)$, the time-sinceinfection dependent coefficient of transmission from an infected farm to susceptible ones $(\beta)$, as well as the spatial kernel $(\rho(\delta))$. The estimation was carried out by 1) designing formulae for the infectiousness and susceptibility of each farm and the farms at a given distance from it as well as for two reproduction numbers ("effective reproduction number" and the "interventionadjusted number"); 2) using various epidemic data from five databases; and 3) using an iterative procedure to determine $\alpha, \tau, \beta$, and $\rho$ by iterating the equations until there was statistical convergence. The authors claim (but do not provide details) that this iterative procedure gave results close to the maximum likelihood estimation using generalized nonlinear multidimensional optimization methods.

In this analysis Ferguson et al. took into consideration several factors that are known to impact infectiousness and susceptibility. Farms were classified into classes according to the number of each animal species; each class has its specific infectiousness. Each farm also was classified according to its susceptibility, which depends on the number of animals as well as on the number of parcels in the farm.

Studying a possible relation between farm susceptibility and farm fragmentation is a unique feature of this model. This is done by assuming that the susceptibility of a farm is linearly proportional to the number of parcels. The estimation of parameters confirmed the positive correlation.

The iterative parameter estimation procedure is the most remarkable feature of this paper but is not explained in detail. It is not pointed how and which data were used in the iterations, nor is there any mention why convergence is guaranteed.

Some of the estimated quantities, such as the "average transmission rate" and the spatial kernel were used to initiate the model from the previously discussed paper. Simulations with the model were used to demonstrate the relative effectiveness of various culling strategies.

\section{Critical remarks.}

Even with improved coefficients, the model does not have predictive value, which is admitted by the authors as well. All previously expressed criticisms for this model still hold.

\subsubsection{Keeling et al. (200I)}

Keeling et al. 2001 developed a spatially-explicit discrete stochastic model of the UK 2001 epidemic. The model uses data on all livestock holdings in the UK from the 2000 census, which includes: location of the farm house, area of farm, number of cattle, sheep, pigs, goats, deer. The individual modeling unit is the farm and the individual states are either susceptible, latent (incubating), infectious, or removed (slaughtered). Transmission between the susceptible and latent class 
incorporates a measure of farm heterogeneities with regard to size and species composition. This is implemented by representing the probability of infection $P_{i}$ of a susceptible farm numbered $i$ as a function of its "susceptibility" and of the "infectiousness" quantities of all infected farms scaled by the distance between them. The susceptibility of farm $i$ is assumed to be a function of the susceptibilities of sheep $S_{s}$ and cattle $S_{c}$ and the quantities of these species $\left(N_{s, i}\right.$ and $\left.N_{c, i}\right)$ on the farm. Similarly the infectiousness of a farm $j$ is a function of virus transmissibility for sheep $T_{s}$ and for cattle $T_{c}$ and the quantities of these species $\left(N_{s, j}\right.$ and $N_{c, j}$ ) on the farm.

$$
\begin{aligned}
P_{i}= & 1-\exp \left[-\left(S_{s} N_{s, i}+S_{c} N_{c, i}\right)\right. \\
& S_{j \in \text { Infectious }} \\
& \left.\left(T_{s} N_{s, j}+T_{c} N_{c, j}\right) K\left(d_{i j}\right)\right]
\end{aligned}
$$

The quantity $S_{s} N_{s, i}+S_{c} N_{c, i}$ is the susceptibility of farm $i$, a linear function of the size of the cattle and sheep herds. $T_{s} N_{s, j}+T_{c} N_{c, j}$ is the infectiousness of farm $j$. The quantity $K\left(d_{i j}\right)$ is the contact kernel and is used to scale down the infectiousness of farm $j$ to a quantity depending on the distance of farm $j$ to farm $i$. Thus, the transmission of infection depends on the following parameters: susceptibilities of sheep and cattle, numbers of sheep and cattle on the susceptible and on the infectious farm and distances between the susceptible farm and the infectious ones.

A random number generator is then used to decide whether the infection occurred, based on this probability.

The probability of farm $i$ not to be infected, $1-P_{i}$, is formulated as the product of the probabilities $\exp \left[-\left(S_{s} N_{s, i}+S_{c} N_{c, i}\right)\left(T_{s} N_{s}+T_{c} N_{c}\right) K\left(d_{i j}\right)\right]$ of farm $i$ to not be infected by any of the infectious farms; i.e. the events of non-infection are considered to be independent of each other.

The transition periods (incubation and infectious) are kept constant at their mean values. The infectious period includes the period from detection to slaughter.

Three types of culling (contiguous, dangerous and $3 \mathrm{~km}$ ) are programmed to simulate the events occurring during the UK 2001 epidemic. Contiguous contacts are defined using a statistic about the average number of neighboring farms because the exact boundaries and neighborhood information is not available. Dangerous contacts were defined as proportional to the infectious contacts by using the contact kernel.

A combination of a maximum likelihood procedure and repeated simulations was used to fit the four parameters (relative susceptibility of cattle compared to sheep, cattle and sheep transmission coefficients and ratio of dangerous to infectious contacts). The authors state that additional least square fits were used to "finetune" the parameters and obtain regional spatial pattern fits. One has to keep in mind that the obtained values of sheep and cattle susceptibility and transmissibility do not represent real estimates.

Both the temporal and spatial patterns of the epidemic were reconstructed quite well by this procedure. It is remarkable that this could be achieved by fitting only four parameter values.

The authors also fit the model while relaxing some of the heterogeneities assumptions. When assuming that all farms are equal (equal herd sizes) and all species are equal, the model could still be fit to mimic the epidemic rather well.

In a later paper Keeling et al. (2003) used the model to model three types of vaccination strategies: mass reactive vaccination, ring vaccination and predictive vaccination. 


\section{Critical remarks.}

1. The basic assumption of this model is that the main factors driving the epidemic are a) the farm sizes, b) the individual abstract "susceptibility" and "transmissibility" of the main affected species, cattle and sheep, and c) the form of the contact kernel. Species susceptibility and transmissibility were not defined explicitly but rather perceived as abstract quantities that need to be identified by fit. The numbers produced by the fit have no practical significance and even real meaning and cannot be used to simulate another epidemic.

2. A fundamental assumption of the model is that the events of the infectious farms infecting a given susceptible farm are independent and defined by the distance between the farms. This assumption is arguable when more attention is paid to the mechanism of transmission. Contact between farms is not defined only by distance but rather by the management practices that connect the farms by means of common personnel, vehicles, markets, etc. Distance does play a role in the transmission process because the quantity of virus decays with distance, being dissipated in the environment, but defining probability of infection based just on distance is a way to ignore heterogeneities due to the human factor.

3. Factors of chance virus dissemination such as airborne transmission or indirect transmission were not attempted to be modeled explicitly. One could assume that these factors are absorbed in the dependence on the number of animals on the farm. Really, the larger the number of animals on the farm, the larger the number of conveyors of indirect virus transmission (operations, personnel, vehicles). Also, the larger the animals on the farm, the greater the chance that some animals will become infected by airborne or wild animal vector transmission. Thus, according to this logic, larger farms are more susceptible.

However, one has to be careful how susceptibility is defined and how farm size enters in the definition. Farm susceptibility $(S)$ is defined by the number of opportunities for obtaining virus a farm has and depends on the quantity $\left(Q_{k}\right)$ of conveyors of indirect virus transmission (of types $\mathrm{k}$ ) as well as on how risky $\left(R_{k}\right)$ each conveyor is. One can write this statement in functional form as

$$
S=F\left(\Sigma_{k} Q_{k} R_{k}\right)
$$

The quantity of conveyors depends on the size of the farm N. For example, depending on the number of cattle, the quantity of milk produced on a dairy farm has to be transported by a certain number of milk collector tankers which visit the farm and probably other farms thus enabling indirect transmission. Thus we can write the above equation as

$$
S=F\left(\Sigma_{k} Q_{k}(N) R_{k}\right)
$$

The dependence of the quantity of indirect contacts or of airborne transmission on farm size might not be linear, especially for large farm sizes. For a large farm, it is less likely that the same vehicles will perform service on other farms or that the same veterinarian, hoofer, etc. personnel will also work for many other similarly large farms. Thus, indirect transmission might actually decrease between farms with increasing farm sizes. This is illustrated on Figure 4. This relation has not been studied and might be worth exploring in the future. If this is the case, farm susceptibility cannot be assumed to be a linear function of farm size. 


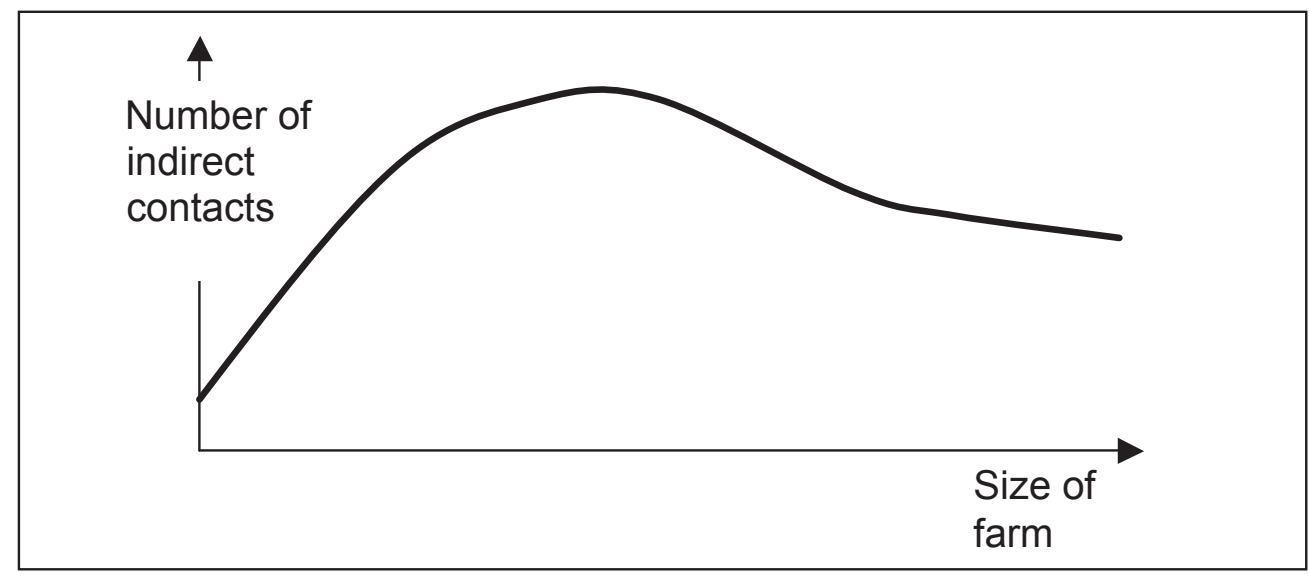

Figure 4. Possible relation between the size of a farm and the quantity of indirect contacts

Therefore, the good spatio-temporal of the model to data may mean that the model of Keeling et al. might work for the UK.

However, it may not be applicable to modeling FMD epidemics in Texas, for example. Again, a good quantitative understanding of the relationship between farm size and the quantity of contacts between farms is the key to determining the scope of applicability of models of that type.

4. A central assumption in this model is that the dynamics of the on-the-farm epidemics does not play a role in the overall epidemics. That is, the quantity of infected animals on any individual farm does not have considerable impact on the overall epidemic speed or proportion. The impact of the number of infectious animals on the farm is again absorbed in the total number of animals. This assumption can hold for comparably small herds and when airborne transmission is not considered, but it might not be true otherwise. This would imply that the model will not be valid for another strain, another season or a different country.

5. Only two species are considered as driving the transmission of infection of this model. Although there were farms containing pigs that were infected and slaughtered in the epidemic, the role of pigs in this epidemic was considered negligible. Probably the reason for not including more species in the model was to have fewer parameters to fit.

6. The definition of contiguous and dangerous contacts in the model is unclear. Although the authors use data for the locations of farms, it does not allow them to define with certainty which are the neighboring farms. Dangerous contacts should be the ones known to have had contacts with an infected farm and not just identified from the contact kernel.

7. Finally, that the model was able to mimic the epidemic after fitting the parameters, does not automatically imply its validity. The fact that a variant of the model in which all farms and all species were considered equal could still fit the temporal dynamics of the epidemic considerably well is disturbing. It means that either the farm and species details are insignificant for the speed and magnitude of the epidemic or that the more detailed model can be fit in more than one way. If this is the case, which set of parameters is the right one? Do different sets give the same predictions regarding the control strategies? The 
uniqueness of the fit was not discussed in the paper.

\subsubsection{Kao (200I, 2003)}

Kao (2001, and 2003) developed a spatial stochastic model that, according to him, represents better spatial heterogeneity and better defines the notion of contiguous farm. The UK map is represented as a hexagonal grid. Each hex contains on average 1.75 premises and contiguous premises of a farm are those that are at 0 or 1 hex distance away. Premises are (somehow) assigned random risk factors $r_{i}$ to account for non-species dependent heterogeneity. The logic of the model is otherwise similar to that of Keeling et al. (2001). The transmission probability from the susceptible to the infected class depends on the contact kernel, the susceptibility of the farm $\theta_{i}$ and the transmissibilities $\tau_{j}$ of the infectious farms. The differences are that the infectiousness of infected farms depends on the time since infection through the proportion of infectiousness $\Delta\left(t_{j}\right)$ on day $j$ and that the probability of non-infection is not taken in the form of an exponential (as in (1) above) but as a linearization of the exponential:

$$
P_{i}=1-\Pi_{j} \in \text { Infectious }\left\{1-\left(R_{0} / 6\right) \Delta\left(t_{j}\right) K\left(d_{i j}\right) \theta_{i} \tau_{j} r_{i} r_{j}\right\}
$$

The rationale behind including the basic reproduction number $R_{0}$ as a factor in the probability that farm $\mathrm{j}$ infects farm $\mathrm{I}$ is not clear. The values of $\theta_{\mathrm{I}}$ and $\tau_{j}$ were taken from Keeling et al. (2001).

This paper makes an important observation that "the best policy is not easily predicted by straightforward assessment of the risk of contact".

\section{Critical remarks.}

All criticisms regarding the model of Keeling and coauthors holds for this paper as well.

The models by Ferguson et al. (2001), Keeling et al. (2001) and Kao (2001) share the common ideology: the transition from the susceptible to the infected herd class is defined as a function of distance and eventually a measure of virus transmission and susceptibility. This approach is more suitable for modeling intra-herd epidemics, where the important factor is close contact. The epidemiology of inter-herd/inter-farm contact is more complex. It does depend on distance-related factors such as airborne transmission that form a baseline, short-range, local spread that can be defined by a distance kernel. However, long-range epidemic spread is mainly effected by direct contacts between farms. The UK modelers did not consider long-range direct contacts because their models were adapted to the 2001 UK epidemic data and all kinds of direct movements between farms were banned. The models discussed next quantify the transmission rates in a different way.

\subsubsection{Sanson (1993) and Morris (200I, 2002)}

Sanson (Sanson 1993) designed the first and only decision-support system EpiMan for emergency planning and response in case of FMD epidemics. In Sanson's words "The purpose of a DSS is to provide a set of tools to help in the interpretation of data ... to grant decision makers an appreciation of the risks implicit in particular decisions and the factors which can be varied to modify those risks".

The first step in designing the system was to complete a study to predict the magnitude of the infected area after the first source had been detected and confirmed. Evaluating the infected area is very important because this is the area where quarantine and strict control observation is forced when an epidemic starts. The study was based on survey data for the number, risk rank and distance of movements on and off an average farm in a representative region for New Zealand. Then a farm - based map was created for the studied region and overlaid on a topographical map of the region. Stochastic simulations of movements from and into the infected farm from 
other farms were performed that corresponded to the types of movements reported in the survey. The study concluded that the affected area might have a radius of dozens of kilometers (an area with radius of $100 \mathrm{~km}$ covered $95 \%$ of all movements).

The decision-support system has four components: a database management system; a knowledge base; a GIS, on the farm model that estimates the quantity of virus produced in time by the farm; and a inter-farm spread model, InterSpread. It also includes an expert system to assign priorities to tracing movements in the case of a real-time epidemic. In additions to the very detailed description of the system in Sanson's dissertation (Sanson 1993), the system has been publicized also in Sanson et al. (1999) and Morris et al. (2001, 2002). The system is by far the most complete tool to deal with in case of epidemics as well as a tool for analysis. The authors claim that it can be used on a PC (in cases of small epidemics) and with hundreds of PCs connected with a server holding the database (in cases of large epidemics). According to the original description (Sanson 1993), the system uses Oracle DBMS Server and client, Arc/Info GIS, Expert System Shell Nexpert Object, 2.0b, S-Plus statistics package, and is programmed in Turbo Pascal 7 and Microsoft C.

The system has been used in the UK 2001 FMD epidemic. It has also been adapted to perform a retrospective modeling of the classical swine fever epidemic in 1997-1998 in the Netherlands (Jalvingh et al. 1999; Nielen et al. 1996).

Because of the large volume of data generated and needed in an emergency, a DSS should use a computerized database system. The EpiMan DBMS integrates data such as property profiles and livestock numbers and stores important information regarding the progress of the disease and holds all the data for the operation of the DSS. The DBMS data and software are stored on a centralized server and allow input and query processes from the client terminals. Users interact with the system via a graphic user's interface. In the course of an epidemic an important part of the client input are the so-called Farm status reports relayed by telephone that include the current state of the farm (infected, slaughtered, disinfected), assessment of the source, etc. Thus the DBMS contains the records of all outbreaks and emergency response.

The knowledge base contains current knowledge for the epidemiology of FMD. It is designed as a growing knowledge system and is continuously updated with new information from literature and occurring epidemics.

The GIS is an important component of the DSS. Because of the spatio-temporal character of all epidemic information, a GIS can be used to display the epidemic data such as infected and at risk farms. The GIS system utilizes the following layers: a) land ownership and management; b) hydrography; c) road network; d) feral animal distribution; e) topography; f) locations of processing plants and saleyards. The GIS is used routinely to display status maps and airborne spread information, to delineate patrol zones, and to assign at-risk farms to patrol veterinarians.

The underlying model is a fully spatial stochastic state transition model. It incorporates "on-thefarm" submodel which calculates the progress of the disease on a farm once it has been defined as infected. The on-the-farm model uses information for the location of the farm and the number of cattle, sheep, pigs, goats; , the initial infection date; the size of the initially infected group; , the number and species of animals with clinical signs and others. The on-the-farm model is a nonspatial stochastic state-transition model embedded in the larger model. The unit on the farm is a cohort of animals. This is a peculiarity of the model dictated 
by veterinarian expert opinion. Each species of the herd is divided in three cohorts that get infected one after the other which means that after twothree incubation periods the whole cohort will be infected. When half of a species cohort is infected, the disease crosses species. Using tables describing the average virus production of each species during the course of the infection, the total virus production of the farm is calculated. This quantity is later used by a plume model to estimate the risk of far away farms to be infected.

Once the current daily status of an infected farm is calculated, the probability of infection of other farms by this farm is estimated. The risks of infection by airborne transmission of virus are calculated by using data are calculated using probabilities from a previous epidemic. The risks of local infection (in a $10 \mathrm{~km}$ radius) by local indirect spread is calculated in a similar way by taking $10 \%$ of the airborne probabilities and disregarding wind direction.

Spread related to movements of animals, vehicles and personnel can be as far as $200 \mathrm{~km}$ away. Movements are categorized as low, high and medium risk. The average number of various movements was estimated by a subjective method performing a survey in several New Zealand regions. Movements to saleyards are also taken into consideration and represent separate risk for transmission. Specific dairy farms movement related to daily milk pickup is also evaluated. Finally, the probability of recrudescence is also included.

The user can direct the program to perform control strategies. These are preventing movements in the affected area (radius $3 \mathrm{~km}$ around an infected premises), slaughter, pre-emptive slaughter, the creation of a vaccination buffer. Pre-emptive slaughter can be performed on a user-defined percentage of the farms. Vaccination zone can be defined by moving the cursor on the screen around the infected premises. All user- defined control zones can be outlined on the screen.

Stevenson (2003) performed estimates of the predictive performance of EpiMan in the UK epidemic. He concluded that the predictions of the total number of infected premises and their spatial distribution were relatively good for a 14 day period ahead, but poor afterwards.

The model goes far in the detail of factors governing FMD infection process. It is hard to imagine any additional information that can be used.

\section{Critical remarks.}

The criticisms concern the way this information is used. The on-the-farm model employs an artificial mechanism of infection by cohorts. This mechanism was found to predict slower epidemic spread especially when the farms are small. The model uses table data for the secretion of virus from each species which might be arguable. However, as the knowledge base of EpiMan grows, these tables will be updated and hopefully, become more reliable. Similarly, the probabilities of transition between the infected states (incubation period, pre-clinical period) were also taken from probability distribution tables which mifght need to be updated. The probabilities of transmission between farms are based on survey data and are fixed predefined values depending on distance from the infected farm but not on the size or species composition of the susceptible farm. Neither is the level of infectiousness of the infected farm taken into consideration when simulating long-distance (above $10 \mathrm{~km}$ ) risk of infection.

InterSpread seemed to consistently overestimate the number of infected premises when applied during the UK epidemic. It is not mentioned how many simulations per study were performed, nor 
how much time does one simulation takes depending on the size of the affected area. Thus, it is hard to establish the computational effectiveness of the model. It is not clear how suitable the system is to perform numerous scenarios simulations in order to compare them.

\subsection{I0 Bates et al. (2003a)}

Bates et al. (2001, 2003a, 2003b, 2003c) developed a spatially-explicit discrete stochastic state transition model which was used to evaluate several control strategies. This model is closest to the model of Sanson (1993) in the sense that it involves a great amount of "veterinary intelligence". The model was parametrized with data from a specific California region characterized by high herd density. The farms were classified in 12 types according to animal species, industrial purpose (beef, dairy) and size (2 to 3 categories) according to the existing census data. Sale yards were also included.

A distinctive feature of the model is the substantial effort undertaken to collect the data by conducting surveys with a large number of veterinarians and technicians working in the region and obtaining expert opinion from FMD epidemiologists worldwide. Some of the data, such as the probability of adequate contact (direct or indirect contact between an infectious and a susceptible herd that would lead to an infection in the susceptible herd) and the half-time of infection of a herd, might be of general validity and could be used for other modeling efforts of purposes. The rest of the data quantified the average quantity of movements into and out of a specific type of farm and the frequency distributions of distances between farms covered by these movements and is probably of regional validity.

The number of newly infected farms is calculated as follows. The expected number of direct and indirect contacts out of each infected farm is multiplied by the probability that the contact is adequate times the proportion of infected animals in the infectious herd. The probability of adequate contact is generated using Monte Carlo sampling from probability distributions constructed from expert opinion survey responses. These products are summed for all infectious farms. The result is the number of daily adequate contacts. Contacts of the infected farms are generated in a random manner. Of these, some will be with infected or depopulated farms. The number of these is subtracted and the remainder is the number of effective contacts.

The susceptible farms are at risk of infection by a weighted random selected process that depends on the risks of exposure via direct or indirect transmission of the farm. This is calculated from the expected number of contacts on the farm, scaled by the farm's distances to all infectious farms. This process is similar in meaning but not in form to the contact kernel in other models discussed here.

The model incorporates spatial and farm heterogeneity as well as three different modes of transmission. The transition times (from latent to subclinical and to clinical stage) were speciesspecific and fitted to data from Burrows (1968).

The goal of the model is to compare the effect of several control strategies: vaccination within several radii from each infected herd and the baseline, dangerous contacts and pre-emptive slaughter strategy (Bates et al. 2003b). Realistic times of delays in applying the strategies were taken into consideration. A corresponding, very detailed benefit-cost analysis of each strategy was performed that included costs for slaughter indemnity, costs for disposal, cleaning, disinfection and vaccination. 
The model was programmed by using commercial software (Visual Basic Microsoft Corp. and BestFit SDK, v.2.0, Palisades Corp., Newfield, NY).

\section{Critical remarks.}

The model can be improved in several areas.

1. A modification of the model including a module simulating airborne transmission would be appropriate. The current model mimics the effect of airborne or other transmission that is more uniformly distributed by weighting the probability of infection via direct and indirect contacts toward nearby farms.

2. The geographical structure, especially the presence of roads could be taken into consideration. The role of roads as vectors of transmission via indirect contact has been hypothesized (Keeling et al. 2001) and some support for this hypothesis has been offered (Rivas 2003). To estimate indirect contact rates, the distance between farms should be measured by the distance traveled on the road network. It has to be noted that none of the reviewed models incorporates road structure.

3. Applying the model to other geographical regions is not automatic. The data concerning numbers and distances of various contacts per farm may not be valid for other regions. A study relating farm size, region size and the mentioned parameters will be of use. If the contact numbers or farms sizes are substantially different, it is quite possible that the comparison between strategies will produce a different result.

4. It appears from the text that the delay from infection to diagnosis in the non-index herds was taken into consideration. Such delays have been shown to have a substantial effect on the outcome of epidemics and on the results from controls.

5. Only two sizes of herds (small and large) play role in the model; additional studies are needed to evaluate the relation between herd size and transmission rate.

6. The model is small-scale. Being stochastic, it requires numerous repetitive simulations which take several hours to complete. Because it was designed to perform one - time comparative estimates of control strategies, speed was not an issue. However, simulations on a larger scale with this type of complexity and detail as in the model or even bigger are not possible unless the model is implemented in a customized software.

\subsection{I. Schoenbaum and Disney (2003)}

Schoenbaum and Disney (2003) developed a discrete stochastic model similar to the one by Garner and Lack. The spatial distribution of farms was randomly generated with three levels of farm density: low, medium, high However, the tool can be initialized with any type of initial herd data (size of herd and location coordinates). Herds did not have a specified species. Three types of herd sizes specific to the three geographical regions were modeled. The disease states were susceptible, latent, infectious, naturally immune, vaccine immune and removed. The transition from the susceptible to the latent state is modeled as a random process depending on the amount of contacts between farms and the probability of infection transfer. Each farm has a certain, predefined amount of contacts of two types (direct and indirect) with other farms. Two such rates of contact are simulated. The probability of infection transfer is constant for direct and indirect contacts. Elements of airborne transmission are simulated as well. 
The transition times between the infected states are randomly generated from triangular probability distributions.

The times to detection of infection were between 1 and 5 days with increasing probability. Thus these times depended on the time since infection. An interesting feature of this model that is different from other models, is the behavioral element: detection times shorten with the development of the epidemic. Another new feature for this model is the simulation of surveillance. The model is linked to an economic model with substantial detail in cost calculation and analysis.

The software was written in Delphi for Windows. It seems to be pretty flexible with respect to input (change of probability distributions, parameters, input). No mention was included regarding the amount of time one simulation takes so it is hard to evaluate whether the tool is applicable for simulations of large outbreaks.

\section{Critical remarks.}

1. All considered cases assume uniformly random spatial distribution of the farms (as shown schematically on Figure 5a). It is questionable whether this assumption is reasonable. In reality, farms are spatially located in aggregated groups (Figure $5 b$ ). Within a group of spatially aggregated farms, the epidemic would develop much faster because of the higher farm density than among the same number of farms uniformly distributed over a larger area (tentatively marked within ovals on Figures $5 a$ and $5 b$ ). If the frequency of contacts between the aggregated groups is sufficiently high, the epidemic may spread over the whole area much faster in the aggregated versus the uniform case. On the other hand, if the rate of contacts between the aggregated groups is relatively low, the speed of the epidemic will be slower in the aggregated versus the uniform case. Thus, the speed of epidemic spread depends on the degree of farm aggregation and on the rate of contacts between groups of farms. Evaluation of strategies performed on a uniform spatial grid may produce quite unreliable results.

2. Two fixed values, taken from literature, for daily indirect and direct contact rates were used. There is no guarantee that the values are realistic and representative for any US region. Using other values will most probably lead to other estimates of control strategies and epidemic cost.

3. All rates are constant and not dependent on farm size. Farm size is expected to be a significant factor for the spread of disease transmission.

4. Assuming that detection becomes possible 1 day after the farm has become infectious is too optimistic. These discrepancies would skew the estimates of the speed of the epidemic and the effectiveness of the control measures.

5. Only one species was considered.

6. The scenarios were of small size epidemics starting with one infectious case.

7. With a non-zero probability of detection on the very first day, the size of the infected area will be very small. 


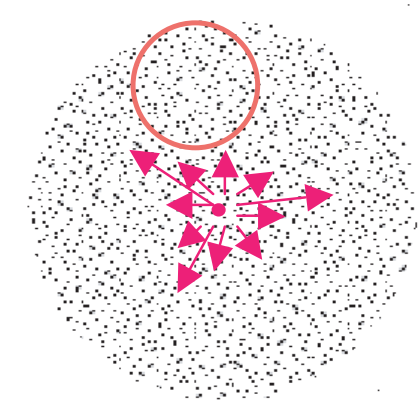

a)

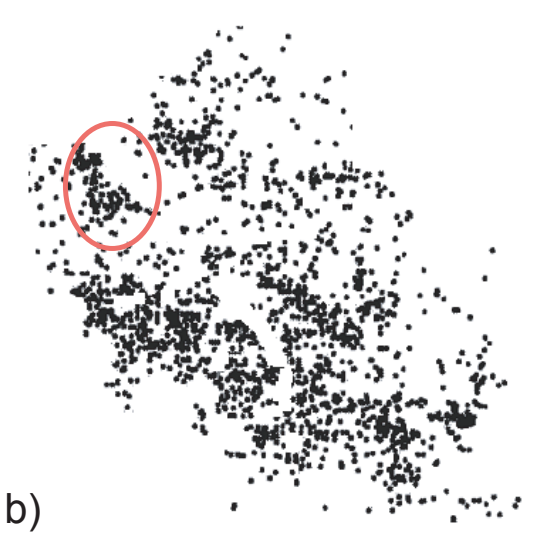

b)

Figure 5. Uniform (a) and aggregated (b) spatial distribution of farms

\subsection{Feature-by-Feature Comparison of All Models}

We have tried to characterize each of the models according to its approach by categorizing them into spatial, non-spatial, top-down and bottom-up and by analyzing their realism. A side-by-side comparison of the specific epidemic factors that each of the models take into consideration can demonstrate their complexity at a glance. Table 1 presents a summary of the features of all models with the exception of the one by Tomassen et al. (2002) which is difficult to assess because of the brevity of exposition of this model. It is obvious that the bottom-up spatial models (Sanson 1993Morris 2001, Bates et al. (2003a) and Schoenbaum and Disney 2003) capture best the complexity of the epidemic process. The complexity of the baseline epidemic modeling of EpiMan (Sanson 1993-Morris 2001) and of Bates et al. (2003a) is comparable and higher than the one of Schoenbaum and Disney 2003.

\subsection{Models Of Airborne Spread.}

Relatively simple models of airborne transmission were included as part of EpiMan (Sanson 1993, Morris 2001, 2002), the model by Tomassen et al. (2001) and the model of Schoenbaum and Disney (2003). Plume models simulating airborne FMDV transmission were also considered by Moutou and
Durand (1994), Cannon and Garner 1999), Sorensen et al. (2000), Donaldson and Alexandersen (2002), Gloster et al. (2003) and Mikelsen et al. (2003).

Detailed analysis of such models is out of the scope of this paper.

\subsection{Other Methods for Analysis of FMDV Epidemics}

\section{I Construction Of Epidemic Trees.}

In the presence of more or less detailed epidemic data, the probable "genealogical" tree structure of the epidemic can be reconstructed with some probability. The construction of the putative epidemic tree allows the estimation of the local (regional) dynamics of the daily basic reproduction numbers and historical analysis of the epidemics. The structure of the epidemic becomes visible and allows the determination of possible isolated sources that should be culled with priority or point to sources which have remained undetected due to mildness of symptoms. 
Table I. The models and their features.

\begin{tabular}{|c|c|c|c|c|c|c|c|c|c|c|}
\hline Properties & $\begin{array}{c}\text { Haydon } \\
\text { et al. } \\
1996\end{array}$ & $\begin{array}{l}\text { Miller } \\
1979\end{array}$ & $\begin{array}{c}\text { Sanson } \\
1993 \\
\text { Morris } \\
2001 \text {, } \\
2002\end{array}$ & $\begin{array}{l}\text { G\&L } \\
1995\end{array}$ & \begin{tabular}{|c|} 
Durand \\
and Mahul \\
2000
\end{tabular} & \begin{tabular}{|c|}
$\begin{array}{c}\text { Ferguson } \\
\text { et al. } \\
200 \mathrm{la}, \\
200 \mathrm{lb}\end{array}$ \\
\end{tabular} & $\begin{array}{l}\text { Keeling } \\
\text { et al. } \\
2001\end{array}$ & $\begin{array}{l}\mathrm{Kao} \\
2001 \\
2003\end{array}$ & $\begin{array}{c}\text { Bates et al. } \\
2001, \\
2003 a, b, c\end{array}$ & $\begin{array}{c}\text { Schoen- } \\
\text { baum and } \\
\text { Disney } \\
2003\end{array}$ \\
\hline $\begin{array}{l}\text { Type of } \\
\text { model }\end{array}$ & $\begin{array}{l}\text { DD } \\
\text { T-D }\end{array}$ & $\begin{array}{l}\text { DD } \\
\text { T-D }\end{array}$ & $\begin{array}{l}\text { SS } \\
\text { B-U }\end{array}$ & $\begin{array}{l}\text { SPS } \\
\text { B-U }\end{array}$ & $\begin{array}{l}\text { DD+SPS } \\
\text { T-D }\end{array}$ & $\begin{array}{l}\text { DODE } \\
\text { T-D }\end{array}$ & $\begin{array}{l}\text { SS } \\
\text { T-D }\end{array}$ & $\begin{array}{l}\text { SS } \\
T-D\end{array}$ & $\begin{array}{l}\text { SS } \\
B-U\end{array}$ & $\begin{array}{l}\text { SPS } \\
\text { B-U }\end{array}$ \\
\hline Time unit & I day & I week & I day & I week & Half week & I day & I day & I day & I day & I day \\
\hline Controls & N/A & S, CS & S,CS, V & $\begin{array}{c}\mathrm{S}, \mathrm{CS} \\
\mathrm{S}+\mathrm{V}\end{array}$ & S,DS,V & CS, V & $\begin{array}{c}\text { S,CS,DS, } \\
\text { PS }\end{array}$ & $\begin{array}{l}\text { S, CS, } \\
\text { DS, V }\end{array}$ & S, DS, PS, V & S, DS, TS \\
\hline $\begin{array}{l}\text { Economic } \\
\text { model }\end{array}$ & No & No & no & yes & yes & no & no & no & yes & yes \\
\hline States & S, L, I, R & $S, I, R, M$ & $\begin{array}{c}S, I, P, R \\
M\end{array}$ & $S, I, R, M$ & $\begin{array}{l}\text { S,E,L,I,P, } \\
\text { M,R }\end{array}$ & $S, E, I, R, X Y$ & S,L,I,R & $S, I, R$ & S,L,I,P,R & S,L,I,M,R \\
\hline $\begin{array}{l}\text { TP depending on } \\
\text { distance }\end{array}$ & $N / A$ & No & yes & no & yes & yes & yes & yes & yes & yes \\
\hline $\begin{array}{l}\text { TP depending on } \\
\text { herd/farm size }\end{array}$ & $\mathrm{N} / \mathrm{A}$ & No & yes & no & yes & no & yes & yes & $\begin{array}{c}\text { Partially } \\
\text { (only two } \\
\text { farm sizes } \\
\text { considered) } \\
\end{array}$ & no \\
\hline $\begin{array}{l}\text { TP depending on } \\
\text { multiple species }\end{array}$ & N/A & No & yes & yes & yes & $\begin{array}{c}200 \text { la no } \\
200 \text { lb yes }\end{array}$ & yes & yes & yes & no \\
\hline $\begin{array}{l}\text { TP depending on } \\
\text { time since } \\
\text { infection }\end{array}$ & $N / A$ & No & yes & no & no & $\begin{array}{c}200 \mathrm{la} \text { no } \\
200 \mathrm{lb} \text { yes }\end{array}$ & no & yes & $\begin{array}{c}\text { Yes, via } \\
\text { intraherd } \\
\text { model }\end{array}$ & no \\
\hline $\begin{array}{l}\text { Species-specific } \\
\text { transition rates }\end{array}$ & N/A & no & yes & no & no & no & no & no & yes & no \\
\hline GIS used & $\mathrm{N} / \mathrm{A}$ & no & yes & no & no & no & no & no & no & no \\
\hline $\begin{array}{l}\text { Intra-herd } \\
\text { transmission }\end{array}$ & N/A & no & yes & no & yes & no & no & no & yes & no \\
\hline $\begin{array}{l}\text { Direct vs. indirect } \\
\text { transmission }\end{array}$ & N/A & no & yes & no & yes & no & no & no & yes & yes \\
\hline $\begin{array}{l}\text { Airborne } \\
\text { transmission }\end{array}$ & N/A & no & yes & no & no & no & no & no & no & yes \\
\hline $\begin{array}{l}\text { Markets } \\
\text { (saleyards) } \\
\text { present }\end{array}$ & $\mathrm{N} / \mathrm{A}$ & no & yes & no & no & no & no & no & yes & no \\
\hline $\begin{array}{l}\text { Transmission } \\
\text { depending on farm } \\
\text { type (beef, dairy) }\end{array}$ & N/A & no & yes & no & no & no & no & no & yes & no \\
\hline $\begin{array}{l}\text { Network } \\
\text { structure }\end{array}$ & N/A & no & implicit & no & no & yes & no & no & no & no \\
\hline
\end{tabular}

\section{Abbreviations:}

Model Types: DD- discrete deterministic state-transition model; DODE - deterministic ordinary equations model, SPS stochastic spatially-uniform state-transition model, SS - spatially-explicit stochastic state transition model; T-D-“top-down”, BU-"bottom-up".

States: $S$ - susceptible, I-infectious, $M$ - immune, $R$ - removed, $X Y$ - pairs of types $X, Y$ where $X, Y$ can take values $S, E, I, R$. Controls: S(slaughter), CS (contact slaughter), DS (dangerous contacts), PS (pre-emptive slaughter), TS (traced contacts), $\mathrm{V}($ vaccination);

TP - transition probability 
Haydon et al. (2003) constructed epidemic trees from data of the 2001 UK epidemic by using three rules for selecting "parent candidates" for each untraced infected premises from a list of possible candidates. It was not possible to determine an epidemic tree in a unique way because some of the rules were probabilistic, 500 trees were constructed and the average properties were examined.

This approach to the analysis of epidemic data is promising as it can be also used in the course of an epidemic as a valuable resource. It is worthwhile to devote efforts for developing and justifying more and better methods for constructing epidemic trees.

\subsection{Visualizing The Dynamics Of The Epidemic.}

In the words of Stevenson 2003, " the 2001 FMD epidemic ... has been a timely reminder of the value of geo-referenced farm data in the management of animal disease outbreaks". Tools that visualize the progress of an epidemic by bringing out internal characteristics of the epidemic such as the regional speed and magnitude of the infection spread, would be essential for evaluating the situation and planning strategies.

Willesmith et al. (2003) describe some spatial analysis methods. One of these, termed "extraction map" plots the ratio of the weekly newly infected cases at the end of the week versus the at risk cases in the beginning of the week for a given spatial region, thus measuring the local speed of the epidemic. Clements et al. (2002) present an interactive atlas of world-scale spatio-temporal presentation, integration and analysis of livestock data.

\subsection{Geographic Information Systems (GIS) And Correlation Analysis}

Geographic Information Systems (GIS) and Correlation Analysis were used by Rivas et al. (2003) "to identify variables associated with epidemic progression". They analyzed retrospectively epidemic data from a Uruguay 2001 outbreak. A remarkable feature of their analysis is the use of the road network via the GIS. Multiscale data was assessed through the GIS: multi-state, state, county and local. County-level data included road density and county area. The local data included point location of infected farms, size of farms and ownership. The authors used the epidemic data from the first 6 days of the epidemic to derive correlations between number of cases, road density, farm density, distance of affected farm to nearest road and other variables. Based on the derived correlations, a tentative region was defined that was assumed to define the direction of epidemic spread. The authors claim that the region contained nearly $90 \%$ of the cases that developed in the next two months. The paper demonstrates that infection spread develops along the road network. The importance of addressing traffic density as a vector of infection was emphasized.

\subsection{Methods For Optimization Applied to Control of FMD}

A simple deterministic SIR model was used by Mathews et al. (2003) to estimate the optimal number of removed premises depending on the proportion of low versus high transmissibility premises. Although the model is quite general and its results are not applicable to a real situation, the paper generates the idea that control measures should be defined by an optimization procedure specific to the regional characteristics.

All approaches used to analyze FMD control strategies compare predefined, with fixed parameters, strategies such as $3 \mathrm{~km}$ cull, $5 \mathrm{~km}$ cull 
and $10 \mathrm{~km}$ vaccination. Optimization methods that find the optimal parameters of a strategy for a given region with specific farm density and species distribution should be able to define the right strategy for the region.

\section{I0.0 Proposed Research and Development}

\section{I Gap Analysis}

We summarized some of the most important conclusions from our review and analysis in Section 3. In addition we note that all considered models lack some important features that should be included in future developments and which we briefly characterize below.

Sensitivity analysis. All simulation models use parameter values either fixed or varying in fixed intervals according to predefined probability distributions. It is important that the model has the capability to test the sensitivity of the simulation results to the parameter values and to the form of the probability distributions.

Qualitative analysis. This is a technique known from the theory of dynamical systems. The idea is that if a model parameter varies in a set of values, the results from the model may be similar over a certain parameter range but completely different in another.

Exploring the different model outcomes gives an idea of the importance of certain parameter value sets.

Road network. All models measure distance between farms as the shortest distance between the points denoting the farms. As the study by Rivas et al. (2003) has shown, the direction of infection spread is rather carried out along the road network system; it may be more realistic to measure distance between farms as the shortest road distance.
Epidemic networks. Complex networks methods might be very useful if a network structure is introduced in the model.

Intra-herd model. The two models that incorporate an intra-herd model (Sanson-Morris and Bates et al. 2003a) use very simple models. A more elaborate intra-herd model will bring more realism and will allow to investigate problems related to farm management optimization (with respect to disease control), farm transmissibility and susceptibility relative to farm size, etc.

Scale. The existing models do not address the problem of scale and none of them has been designed to handle large-scale computations. There are two important issues that require the development of fast-speed, large-scale computational epidemic models. First, the US scale of herd sizes and number of herds is considerably larger than these of other countries where modeling has been attempted. Second, the stochastic nature of the epidemic data requires the use of a stochastic model and numerous (hundreds) of repetitive simulations to obtain a statistically reliable result.

\subsection{Proposed Research}

\subsection{The Grand Challenge: Development of a Decision-Support System}

A decision-support system can play a very significant role in the event of a bioterrorist attack in the agricultural sector. It can assist decision makers in planning the nature, location, and time of measures for response so that the expected losses be minimized. Such a system ideally would:

- Rely on various data sources, such as

- spatial locations of US farms;

- types and sizes of US herds;

- actual pasture lands used; 
- a network structure of the personnel working on each farm, of farm ownership and common transportation vehicles used;

- evaluations of intra-herd epidemic contact data;

- specifics regarding the management of farms that influence the epidemic process;

- specific epidemic data, including strainspecific data.

- Represent a complex computational model consisting of a baseline epidemic model with superimposed strategic models representing the (currently available) methods of epidemic control (vaccination, slaughter, PCR detection, contact tracing, etc.) and connected to a module for cost-benefit analysis.

The baseline epidemic model should

- be large-scale and fast-speed in order to accommodate two needs: applicability on the US scale and capability to perform a large number of repetitive stochastic simulations;

- $\quad$ be open-ended, i.e., easily modifiable to include new data sources or replace less accurate submodels with improved ones;

- incorporate a "good" intra-herd model (including management types, age- and species-specific disease modeling, reliable contact estimates);

- be spatially explicit to take into account the spatial density heterogeneity of farm distribution and farm aggregation;

- utilize a geographic information system to facilitate visualization and fast orientation in the epidemic situation;

- represent the known network structure of farm contacts (each farm belongs to several different networks connecting it with farms serviced by the same veterinarian or the same insemination technician, restocked at the same saleyards, or served by the same dairy trucks; the affiliation of a farm with a network defines the possible routes of indirect infection);

- take into consideration all identified above transmission factors between farms (speciesspecificity, density dependence, distance dependence, farm fragmentation, airborne transmission, road network);

- be complemented with tools for:

- evaluation of the initial infected area prior to the detection of the first case;

- discrete optimization (to perform searches for best strategies);

- sensitivity analysis (to perform estimates of the significance of specific data accuracy;

- determination of the index case by a combination of matching criteria;

- visualization of the progress of the epidemic and of the areas where control is applied.

\subsubsection{Basic Research to Support the Development of a Decision Support System}

Obviously, it cannot be expected that such a system can be built in the "heat of an epidemic." First, most of the required data does not exist or is not complete or sufficiently accurate. Second, the construction of a computational model of this complexity requires considerable time and team effort.

However, the good news is that the construction of a computational model with the desired qualities can be performed without the actual data. The work on the model can be done while the data is collected. In the meantime, there are computational and modeling issues that need to be addressed, such as

- Developing in-depth expertise in large-scale spatially explicit epidemic models. The model 
of Bates et al. (2003a) can serve as a template for developing a parallel version;

- Construction of methods to develop "adaptively-predictive" models; i.e., models that improve their predictive accuracy upon use of newly arriving data;

- Research to establish the limitations of simulations with uniform versus aggregated farm spatial data;

- Development of efficient optimization techniques for the purpose of fast determination of optimal strategic responses. All the existing models follow the same routine to compare several predefined strategies. However, depending on the studied region, distribution of farms, etc. it may be impossible to determine in advance which strategies to compare. The correct, though computationally more expensive procedure, is to search for the optimal strategy. For example, search for the radius of the vaccination ring that guarantees minimal loss and economic cost;

- Fast, efficient methods for sensitivity analysis of agent-based models;

- Exploration of methods to combine agentbased models and network models. Each farm can be considered as an element of several networks that define the contacts between the farm and other farms. For example farms served by the same insemination technician form a network. The probability of transmission along the network is higher than outside of it. If the networks are known, the spread of infection can be predicted with a great probability. Here the theory of complex networks would be of help.

The successful resolution of these issues will be of help in building other spatial models such as largescale urban epidemic models.

\subsubsection{Smaller, Short-Term Tasks}

A list of possible smaller, independent from each other, topics of research and development is proposed next.

Intra-herd modeling. The development of more detailed intra-herd models will contribute to understanding the dynamics of FMD on an individual farm. The specifics of the farm management practices should be considered and the question what farm organization and production schedule minimizes disease spread should be addressed. Farms with different types of management should be modeled and the scope of spread of simulated epidemics should be analyzed and compared. Such exercises can lead to alteration of management practices to ensure lowest risk of disease transmission in case of an outbreak. A study of the existing farming practices should be conducted to supply initial basis for the model.

Size of initial infected area. All epidemics are different. Models to evaluate the maximum size of an infected area at the start of an epidemic based on information at the time of detection of the first case would be very useful in the case of an outbreak. They will allow to plan the necessary resources for containing the epidemic from the very start. The formulation of such a model will identify the necessary data that needs to be available as a precautionary measure.

Risk maps. A ready tool to produce spatial US maps of highest risk will be very useful in case of epidemic outbreak.

Epidemic trees. Software implementing the construction of epidemic trees to estimate the sources of the outbreak would be another useful tool in case of outbreak. 


\section{References}

Alexandersen S, Donaldson AI, 2002, Further studies to quantify the dose of natural aerosols of foot-and-mouth disease virus for pigs, Epidemiology And Infection, 128 (2): 313-323

Alexandersen S, Zhang Z, Reid SM, Hutchings GH, Donaldson AI 2002a, Quantities of infectious virus and viral RNA recovered from sheep and cattle experimentally infected with foot-and-mouth disease virus O UK 2001, Journal Of General Virology, 83: 1915-1923 Part 8

Alexandersen S, Brotherhood I, Donaldson AI, $2002 b$, Natural aerosol transmission of foot-andmouth disease virus to pigs: minimal infectious dose for strain O-1 Lausanne, Epidemiology And Infection, 128 (2): 301-312

Alexandersen, S., Zhang, Z., Donaldson A.I., Garland, A.J.M., 2003a, The Pathogenesis and Diagnosis of Foot-and-Mouth Disease, J. Comp. Path., 129, 1-36

Alexandersen, S., Quan, M., Murphy, C., Knight J., Zhang, Z., 2003b, Studies of Quantitative Parameters of Virus Excretion and Transmission in Pigs and Cattle Experimentally Infected with Footand-Mouth Disease Virus, J. Comp. Path., 129: 268282

Bates TW, Carpenter TE, Thurmond MC. 2003 Epidemiologic information for modeling foot-andmouth disease. Bioterrorism: Mathematical Modeling Applications in Homeland Security (Invited book chapter). Society for Industrial and Applied Mathematics (SIAM) pg 111-132..

Bates TW, Thurmond MC, Carpenter TE, 2001a, Direct and indirect contact rates among beef, dairy, goat, sheep, and swine herds in three California counties, with reference to control of potential foot-and-mouth disease transmission, American Journal Of Veterinary Research, 62 (7): 1121-1129

Bates TW, Thurmond MC, Carpenter TE, 2003a, Description of an epidemic simulation model for use in evaluating strategies to control an outbreak of foot-and-mouth disease, American Journal Of Veterinary Research, 64 (2): 195-204

Bates TW, Thurmond MC, Carpenter TE, 2003b, Results of epidemic simulation modeling to evaluate strategies to control an outbreak of footand-mouth disease, American Journal Of Veterinary Research, 64 (2): 205-210 Feb 2003

Bates TW, Carpenter TE, Thurmond MC, 2003c, Benefit-cost analysis of vaccination and preemptive slaughter as a means of eradicating foot-and-mouth disease, AMERICAN Journal Of Veterinary Research, 64 (7): 805-812 JUL 2003

Bouloux C, Langlais M, Silan P, 1998, A marine host-parasite model with direct biological cycle and age structure, Ecological Modelling, 107 (1): 73-86

Bouma A, Elbers ARW, Dekker A, de Koeijer A, Bartels C, Vellema P, van der Wal P, van Rooij EMA, Pluimers FH, de Jong MCM, 2003, The foot-andmouth disease epidemic in The Netherlands in 2001, Preventive Veterinary Medicine, 57 (3): 155-166

Burrows R., Excretion of foot and mouth disease virus prior to the development of lesions, Vet. Rec., 82: $387-388$

Cannon RM, Garner MG, 1999, Assessing the risk of wind-borne spread of foot-and-mouth disease in Australia, Environment International, 25 (6-7): 713723

Carpenter T., Thieme A., A Simulation Approach to Measuring the Economic Effects of Foot-andMouth Disease in Beef and Dairy Cattle, 1980, Proc. $2^{\text {nd }}$ International Symposium on Veterinary Epidemiology and Economics, Canberra, Australia, 7-11 May1979, 511-516

Carpenter T., Thurmond M., Bates T., A Simulation Model of Intraherd Transmission of Foot and Mouth Disease with Reference to disease spread before and after clinical diagnosis, 2004, J. Vet. Diagn. Invest., 16:11-16

Clements ACA, Pfeiffer DU, Otte MJ, Morteo K, Chen L, 2002, A global livestock production and health atlas (GLiPHA) for interactive presentation, integration and analysis of livestock data, Prev Vet Med 56 (1): 19-32

Domingo, E., Escarmis, C., Baranowski, E., RuizJarabo, C.M., Carrillo, E., Nunez, J.I., Sobrino, F., (2003) , Evolution of foot-and-mouth disease virus, Virus Research 91: 47-63 
Donaldson AI, Alexandersen S, 2002, Predicting the spread of foot and mouth disease by airborne virus, Revue Scientifique Et Technique De L Office International Des Epizooties, 21 (3): 569-575

Durand B, Mahul O., 2000, An extended statetransition model for foot-and-mouth disease epidemics in France, Preventive Veterinary Medicine, 47 (1-2): 121-139

Feng Z, Iannelli M, Milner FA, 2002, A two-strain tuberculosis model with age of infection, SIAM J. Appl. Math., 62 (5): 1634-1656

Ferguson NM, Donnelly CA, Anderson RM, (2001a) The foot-and-mouth epidemic in Great Britain: Pattern of spread and impact of interventions, Science, 292 (5519): 1155-1160

Ferguson, N.M., Donnelly, C.A., Anderson, R.M , 2001b., Transmission intensity and impact of control policies on the foot and mouth epidemic in Great Britain, Nature, 413:542-548

Gani R, Leach S, 2001,Transmission Potential Of Smallpox In Contemporary Populations, Nature, 414 (6865): 748-751

Garner M.G., Lack M.B.,1995, An Evaluation of Alternate Control Strategies for Foot and Mouth Disease in Australia: a regional approach,

Preventive Veterinary Medicine 23: 9-32

Gerbier G, Bacro JN, Pouillot R, Durand B, Moutou F, Chadoeuf J, 2002, A point pattern model of the spread of foot-and-mouth disease, Preventive Veterinary Medicine, 56 (1): 33-49

Gloster J, Champion HJ, Sorensen JH, Mikkelsen T, Ryall DB, Astrup P, Alexandersen S, Donaldson AI, 2003, Airborne transmission of foot-and-mouth disease virus from Burnside Farm, Heddonon-theWall, Northumberland, during the 2001 epidemic in the United Kingdom, Veterinary Record, 152 (17): 525-533

Green, L.E. , Medley, G. F., 2002, Mathematical modelling of the foot and mouth disease epidemic of 2001: strengths and weaknesses, Research in Veterinary Science, 73, 201-205

Grubman MJ, Baxt B., 2004, Foot and Mouth Disease, Clinical Microbiology Reviews, 17(2): 465493
Haydon D.T., Woolhouse M.E.J., Kitching R.P., 1997, An analysis of foot-and-mouth-disease epidemics in the UK, IMA Journal Of Mathematics Applied In Medicine And Biology, 14 (1): 1-9

Haydon, D. T., Chase-Topping, M., Shaw, D. J., Matthews, L., Friar, J. K., Wilesmith, J., Woolhouse, M. E. J., 2003, The construction and analysis of epidemic trees with reference to the 2001 UK footand-mouth outbreak, Proc. R. Soc. Lond. B, 270, 121-127

Hughes GJ, Kitching RP, Woolhouse MEJ, 2002, Dose-dependent responses of sheep inoculated intranasally with a type $\mathrm{O}$ foot-and-mouth disease virus, Journal Of Comparative Pathology, 127 (1):22-29

Hutber A.M., Kitching R.P., (1996), The use of vector transition in the modelling of intraherd foot-and-mouth disease, Environmental And Ecological Statistics, 3 (3): 245-255

Jalvingh AW, Nielen M, Maurice H, Stegeman AJ, Elbers ARW, Dijkhuizen AA, 1999, Spatial and stochastic simulation to evaluate the impact of events and control measures ion the 1997-1998 classical swine fever epidemic in The Netherlands. I. Description of simulation model, Preventive Veterinary Medicine, 42 (3-4): 271-295

Kao RR, 2001, Landscape fragmentation and footand-mouth disease transmission, Veterinary Record, 148 (24): 746-747

Kao, R.R., 2002, The role of mathematical modelling in the control of the 2001 FMD epidemic in the UK, Trends in Microbiology, Vol.10 No.6

Kao, R.R., 2003, The impact of local heterogeneity on alternative control strategies for foot-andmouth disease, Proc. R. Soc. Lond. B, 270: 2557-2564

Keeling, MJ, 1999, The effects of local spatial structure on epidemiological invasions, Proc. Royal Soc. London Series B, 266:859-867

Keeling, M.J., Woolhouse, M.E.J., Shaw, D.J., Matthews, L., Chase-Topping, M., Haydon, D.T., Cornell, S.J., Kappey, J., Wilesmith, J.,Grenfell, B.T., 2001, Dynamics of the 2001 UK Foot and Mouth Epidemic: Stochastic Dispersal in a Heterogeneous Landscape, Science, 294:813-817 
Keeling, M. J. , Woolhouse, M. E. J., May, R.M., Davies G. , Grenfell, B.T. , 2003, Modelling vaccination strategies against foot-and-mouth disease, Nature 421:136-142

Kretzschmar M., Morris M., 1995, Measures of Concurrency in networks and the spread of infectious disease, Math. Biosci., 133:165-195

Leforban Y, Gerbier G ,2002, Review of the status of foot and mouth disease and approach to control/eradication in Europe and Central Asia, Revue Scientifique Et Technique De L Office International Des Epizooties, 21 (3): 477-492

MacKenzie K, Bishop SC, 2001a, Developing stochastic epidemiological models to quantify the dynamics of infectious diseases in domestic livestock, Journal Of Animal Science , 79 (8): 20472056

MacKenzie K, Bishop SC, 2001b, Utilizing stochastic genetic epidemiological models to quantify the impact of selection for resistance to infectious diseases in domestic livestock Journal Of Animal Science , 79 (8): 2057-2065

Mahul O., Durand B., 2000, Simulated economic consequences of foot-and-mouth disease epidemics and their public control in France, Prev. Vet. Med., 47, 23-38

Martcheva M, Castillo-Chavez C, 2003, Diseases with chronic stage in a population with varying size, Mathematical Biosciences, 182 (1): 1-25

Mikkelsen T, Alexandersen S, Astrup P, Champion HJ, Donaldson AI, Dunkerley FN, Gloster J, Sorensen JH, Thykier-Nielsen S, 2003, Investigation of airborne foot-and-mouth disease virus transmission during low-wind conditions in the early phase of the UK 2001 epidemic, Atmospheric Chemistry And Physics, 3: 2101-2110

Miller, W.M. (1979). A state-transition model of epidemic foot-and-mouth disease. In E.H. McCauley, J.C. New, N.A. Aulaqi, W.B. Sundquist and W.M. Miller, A Study of the Potential Economic Impact of Foot-and-Mouth Disease in the United States, University of Minnesota, St. Paul, Minnesota, 113-131.

Morris RS, Wilesmith JW, Stern MW, Sanson RL, Stevenson MA, 2001, Predictive spatial modelling of alternative control strategies for the foot-andmouth disease epidemic in Great Britain, 2001, Veterinary Record , 149 (5): 137-144

Morris RS, Sanson RL, Stern MW, Stevenson M, Wilesmith JW, 2002, Decision-support tools for foot and mouth disease control, Revue Scientifique Et Technique De L Office International Des Epizooties, 21 (3): 557-567

Moutou F., (2002), Epidemiological basis useful for the control of foot-and-mouth disease, Comparative Immunology Microbiology And Infectious Diseases, 25 (5-6): 321-330

Moutou F, Durand B. Modelling the spread of footand-mouth disease virus, 1994, Veterinary Research, 25 (2-3): 279-285

Nielen M, Jalvingh AW, Horst HS, Dijkhuizen AA, Maurice $\mathrm{H}$, Schut $\mathrm{BH}$, vanWuijckhuise LA, deJong MF, 1996, Quantification of contacts between Dutch farms to assess the potential risk of footand-mouth disease spread, Preventive Veterinary Medicine, 28 (3): 143-158

Noordhuizen, J.P.T.M. et al., 2001, Application of quantitative methods in veterinary epidemiology, Wageningen Pers, Wageningen

Rivas AL, Smith SD, Sullivan PJ, Gardner B, Aparicio JP, Hoogesteijn AL, Castillo-Chavez C., (2003), Identification of geographic factors associated with early spread of foot-and-mouth disease, American Journal Of Veterinary Research, 64 (12): 1519-1527

Rosa R, Pugliese A, Villani A, Rizzoli A., 2003, Individual-based vs. deterministic models for macroparasites: host cycles and extinction, Theoretical Population Biology, 63 (4): 295-307 JUN 2003

Royal Society, 2002, “Infectious Diseases in Livestock", The Royal Society policy document 19/02, July 2002, www.royalsoc.ac.uk

Sanson RL, (1993) The development of a decision support system for an animal disease emergency, $\mathrm{PhD}$ thesis, Massey University, Massey

Sanson RL, Morris RS, Stern MW, 1999, EpiMANFMD: a decision support system for managing epidemics of vesicular disease, Revue Scientifique 
Et Technique De L Office International Des Epizooties, 18 (3): 593-605

Schoenbaum, M.A., Disney, W.T., 2003 Modeling alternative mitigation strategies for a hypothetical outbreak of foot-and-mouth disease in the United States, Preventive Veterinary Medicine 58 25-52

Sorensen JH, Mackay DKJ, Jensen CO, Donaldson AI, 2000, An integrated model to predict the atmospheric spread of foot-and-mouth disease virus, Epidemiology And Infection, 124(3): 577-590

Stevenson MA, 2003, The Spatio-temporal Epidemiology of Bovine Spongiform Encephalopathy and Foot-and-Mouth Disease in Great Britain, Thesis, Massey University

Sutmoller, P., Barteling, S.S., Olascoaga, R.C., Sumption, K.J., 2003, Control and eradication of foot-and-mouth disease, Virus Research 91:101-144
Taylor, N., 2003 Review of the use of models in informing disease control policy development and adjustment, A report for DEFRA, http://www.defra.gov.uk/science/Publications/2 003/UseofModelsinDiseaseControlPolicy.pdf

Tinline A., 1982, A simulation study of the 19671968 foot and mouth epizootic in Great Britain, PhD Thesis, University of Bristol

Wilesmith JW, Stevenson MA, King CB, Morris RS, 2003, Spatio-temporal epidemiology of foot-andmouth disease in two counties of Great Britain in 2001, Preventive Veterinary Medicine, 61 (3): 157170

Wilson TM, Gregg DA, King DJ, Noah DL, Perkins LEL, Swayne DE, Inskeep II, W., (2001), Agroterrorism, biological crimes, and biowarfare targeting animal agriculture - The clinical, pathologic, diagnostic, and epidemiologic features of some important animal diseases, Clinics in Laboratory Medicine 21 (3): 549-591 